\title{
Mean value theorems for long Dirichlet polynomials and tails of Dirichlet series
}

by

D. A. Goldston (San Jose, Calif.) and S. M. Gonek (Rochester, N.Y.)

We obtain formulas for computing mean values of Dirichlet polynomials that have more terms than the length of the integration range. These formulas allow one to compute the contribution of off-diagonal terms provided one knows the correlation functions for the coefficients of the Dirichlet polynomials. A smooth weight is used to control error terms, and this weight can in typical applications be removed from the final result. Similar results are obtained for the tails of Dirichlet series. Four examples of applications to the Riemann zeta-function are included.

1. Introduction and statement of results. Let $\left\{a_{n}\right\}_{n=1}^{\infty}$ be a sequence of real or complex numbers such that for any $\varepsilon>0, a_{n} \ll_{\varepsilon} n^{\varepsilon}$ as $n \rightarrow \infty$. Let $s=\sigma+i t$ be a complex variable and let

$$
\mathbb{A}(s)=\sum_{n \leq N} a_{n} n^{-s}
$$

be a Dirichlet polynomial. By Montgomery and Vaughan's mean value theorem [4] we have

$$
\int_{0}^{T}|\mathbb{A}(s)|^{2} d t=\sum_{n \leq N}\left|a_{n}\right|^{2} n^{-2 \sigma}(T+O(n)) .
$$

It immediately follows that if $N=o(T)$ as $T \rightarrow \infty$, then

$$
\int_{0}^{T}|\mathbb{A}(s)|^{2} d t \sim T \sum_{n \leq N}\left|a_{n}\right|^{2} n^{-2 \sigma} .
$$

On the other hand, if $N \gg T$ and $\sigma<1$, the $O$-terms in (1) can dominate so that we lose the asymptotic formula. The situation is similar for the

1991 Mathematics Subject Classification: Primary 11M06; Secondary 11M26.

The work of both authors was partially supported by grants from NSF. 
mean-square of the tail Dirichlet series

$$
\mathbb{A}^{*}(s)=\sum_{n>N} a_{n} n^{-s}
$$

when $\sigma>1$. Our purpose in this paper is to determine the mean-square behavior of $\mathbb{A}(s)$ and $\mathbb{A}^{*}(s)$ even when $N$ is significantly larger than $T$.

If we square out and integrate termwise in (1), we see that the $O$-terms on the right-hand side come from off-diagonal terms. It is these we must carefully estimate therefore when $N$ is large. We treat them by appealing to good uniform estimates for the coefficient correlation functions

$$
A(x, h)=\sum_{n \leq x} a_{n} \bar{a}_{n+h} .
$$

Such estimates are available for $a_{n} \equiv 1, a_{n}=d(n)$ (the divisor function), $a_{n}=\mu^{2}(n)$ (the square of the Möbius function), when $a_{n}$ is the $n$th Fourier coefficient of a modular form, and for a number of other arithmetical functions. Moreover, it is interesting to note that we can often formulate a conjectural estimate for $A(x, h)$ even when we cannot estimate $A(x, h)$ rigorously. In such cases we can then use our theorems to deduce conditional mean value formulae for the associated Dirichlet series.

Since it is no more difficult to treat the more general means

$$
\int_{0}^{T} \mathbb{A}(s) \overline{\mathbb{B}(s)} d t
$$

and

$$
\int_{0}^{T} \mathbb{A}^{*}(s) \overline{\mathbb{B}^{*}(s)} d t
$$

where $\mathbb{B}(s)=\sum_{n \leq N} b_{n} n^{-s}$ and $\mathbb{B}^{*}(s)=\sum_{n>N} b_{n} n^{-s}$, we shall do so.

The precise assumptions we shall make about the sequences $\left\{a_{n}\right\}_{n=1}^{\infty}$ and $\left\{b_{n}\right\}_{n=1}^{\infty}$ are:

$\left(\mathrm{A}_{1}\right)$ For every $\varepsilon>0$ we have $a_{n}, b_{n} \ll_{\varepsilon} n^{\varepsilon}$. write

$\left(\mathrm{A}_{2}\right)$ If $A(x)=\sum_{n \leq x} a_{n}$ and $B(x)=\sum_{n \leq x} b_{n}$, then for $x \geq 0$ we may

$$
A(x)=M_{1}(x)+E_{1}(x)
$$

and

$$
B(x)=M_{2}(x)+E_{2}(x)
$$

where

$$
\begin{gathered}
M_{1}^{\prime}(x), M_{2}^{\prime}(x) \ll_{\varepsilon}(x+1)^{\varepsilon}, \\
M_{1}^{\prime \prime}(x), M_{2}^{\prime \prime}(x) \ll_{\varepsilon}(x+1)^{\varepsilon-1}
\end{gathered}
$$


and

$$
E_{1}(x), E_{2}(x) \ll(x+1)^{\theta}
$$

for some $\theta \in[0,1)$.

$\left(\mathrm{A}_{3}\right)$ The coefficient correlation functions

$$
C_{1}(x, h)=\sum_{n \leq x} a_{n} \bar{b}_{n+h} \quad \text { and } \quad C_{2}(x, h)=\sum_{n \leq x} b_{n} \bar{a}_{n+h}
$$

are of the form

$$
C_{i}(x, h)=M_{i}(x, h)+E_{i}(x, h) \quad(i=1,2)
$$

for $x \geq 0$, where $M_{i}(x, h)(i=1,2)$ is twice differentiable for each $h=$ $1,2, \ldots$, and

$$
E_{i}(x, h) \ll(x+1)^{\varphi} \quad(i=1,2)
$$

uniformly for $1 \leq h \leq x^{\eta}$ for some $\varphi \in[0,1)$ and some $\eta \in(0,1)$.

Sometimes we shall also assume

$\left(\mathrm{A}_{4}\right)$ For every $\varepsilon>0$ we have

$$
M_{i}^{\prime}(x, h) \ll_{\varepsilon} h^{\varepsilon}(x+1)^{\varepsilon} \quad(i=1,2)
$$

uniformly for $x \geq 0$ and $h=1,2, \ldots$

Instead of estimating (2) and (3) directly, we find it more advantageous to estimate the integrals

$$
\text { (11) } I=\int_{-\infty}^{\infty} \Psi_{U}\left(\frac{t}{T}\right)\left(\mathbb{A}(s)-\int_{1}^{N} M_{1}^{\prime}(x) x^{-s} d x\right) \overline{\left(\mathbb{B}(s)-\int_{1}^{N} M_{2}^{\prime}(x) x^{-s} d x\right)} d t
$$

and

(12) $I^{*}=\int_{-\infty}^{\infty} \Psi_{U}\left(\frac{t}{T}\right)$

$$
\times\left(\mathbb{A}^{*}(s)-\int_{N}^{\infty} M_{1}^{\prime}(x) x^{-s} d x\right) \overline{\left(\mathbb{B}^{*}(s)-\int_{N}^{\infty} M_{2}^{\prime}(x) x^{-s} d x\right)} d t .
$$

Here $M_{1}(x)$ and $M_{2}(x)$ are as in (4) and (5) and $\Psi_{U}(t)$ is a real-valued weight function satisfying the following conditions. Let $B>0, U \approx(\log T)^{B}$, and $C_{1} \leq C_{2}$, where $C_{1}$ and $C_{2}$ are bounded but may be functions of $U$. Then $\Psi_{U}(t)$ is supported on $\left[C_{1}-U^{-1}, C_{2}+U^{-1}\right]$,

$$
\Psi_{U}(t)=1 \quad \text { if } C_{1}+U^{-1} \leq t \leq C_{2}-U^{-1},
$$

and

$$
\Psi_{U}^{(j)}(t) \ll U^{j} \quad \text { for } j=0,1, \ldots
$$


(Note that (13) is vacuous if, for example, $C_{1}=C_{2}$.) The removal of $\Psi_{U}$ from $I$ and $I^{*}$ is usually straightforward and will be demonstrated in the examples at the end of the paper.

Before stating our results we introduce some more notation and useful estimates. We use $\varepsilon$ to represent an arbitrarily small positive number which is fixed during the course of each proof. We then set

$$
\tau=T^{1-\varepsilon} \text {. }
$$

We always assume that $\sigma$, the real part of $s$, is bounded above and below. The constants implied by the symbols $O$ and « may depend on $\varepsilon$, the upper and lower bounds for $\sigma$, and other parameters, but never on $T$ or parameters dependent on $T$, like $N$ and $\tau$. Thus, in particular, our $O$-terms hold uniformly for bounded $\sigma$.

We define the Fourier transform of $\Psi_{U}(t)$ by

$$
\widehat{\Psi}_{U}(\xi)=\int_{-\infty}^{\infty} \Psi_{U}(t) e(\xi t) d t
$$

where $e(x)=e^{2 \pi i x}$. It follows easily that

$$
\int_{-\infty}^{\infty} \Psi_{U}\left(\frac{t}{T}\right) e(\xi t) d t=T \widehat{\Psi}_{U}(T \xi)
$$

and, since $\Psi_{U}(t)$ is real, that

$$
\widehat{\Psi}_{U}(-\xi)=\widehat{\Psi}_{U}(\xi)
$$

Observe that $\widehat{\Psi}_{U}$ and $\widehat{\Psi}_{U}^{\prime}$ are trivially $\ll C_{2}-C_{1}+U^{-1}$. Also, integrating by parts $j$ times and using $(14)$, we see that $\widehat{\Psi}_{U}(\xi)$ and $\widehat{\Psi}_{U}^{\prime}(\xi)$ are $\ll\left(C_{2}-\right.$ $\left.C_{1}+U^{-1}\right)(U /(2 \pi \xi))^{j}$ if $\xi \neq 0$. Thus, for $j$ arbitrarily large we have

$$
\widehat{\Psi}_{U}(\xi), \widehat{\Psi}_{U}^{\prime}(\xi) \ll\left(C_{2}-C_{1}+U^{-1}\right) \min \left(1,(U /(2 \pi \xi))^{j}\right) .
$$

It follows that

$$
\widehat{\Psi}_{U}(\xi) \ll \xi^{-D_{1}}
$$

for $\xi \gg T^{\varepsilon}$ and that

$$
\widehat{\Psi}_{U}(T \xi) \ll T^{-D_{2}}
$$

for $\xi \gg \tau^{-1}$, where $D_{1}$ and $D_{2}$ are arbitrarily large constants.

We write

$$
K_{\sigma}(x, u)=K_{\sigma}(x, u, T, U)=x^{-2 \sigma}\left(1+\frac{u}{x}\right)^{-\sigma} \widehat{\Psi}_{U}\left(\frac{T}{2 \pi} \log \left(1+\frac{u}{x}\right)\right)
$$

and easily find by (17) that

$$
K_{\sigma}(x, u) \ll x^{-2 \sigma}
$$


and

$$
\frac{\partial}{\partial x} K_{\sigma}(x, u) \ll x^{-2 \sigma-1} T^{\varepsilon}
$$

for $u / x \ll \tau^{-1}$. By the mean value theorem of differential calculus and (17) we have

$$
\widehat{\Psi}_{U}\left(\frac{T}{2 \pi} \log \left(1+\frac{u}{x}\right)\right)=\widehat{\Psi}_{U}\left(\frac{u T}{2 \pi x}\right)+O\left(T^{-1+2 \varepsilon}\right)
$$

when $u / x \ll \tau^{-1}$. Using this and (17) it is not difficult to deduce the approximation

$$
K_{\sigma}(x, u)=x^{-2 \sigma} \widehat{\Psi}_{U}\left(\frac{u T}{2 \pi x}\right)+O\left(x^{-2 \sigma} T^{-1+2 \varepsilon}\right)
$$

for $u / x \ll \tau^{-1}$.

We can now state our main results.

Theorem 1. Let $\sigma_{1}<\sigma_{2}<1$, let $0<\varepsilon<1 / 2$ be fixed, and let $\tau=T^{1-\varepsilon}$. Suppose that the sequences $\left\{a_{n}\right\}_{n=1}^{\infty}$ and $\left\{b_{n}\right\}_{n=1}^{\infty}$ satisfy $\left(\mathrm{A}_{1}\right),\left(\mathrm{A}_{2}\right)$, and $\left(\mathrm{A}_{3}\right)$ and that

$$
\tau \ll N \leq \tau^{1 /(1-\eta)},
$$

where $\eta$ is as in $\left(\mathrm{A}_{3}\right)$. Set

$$
H=N /(\tau+1)
$$

Then

$$
\begin{aligned}
(26) I= & \int_{-\infty}^{\infty} \Psi_{U}\left(\frac{t}{T}\right)\left(\mathbb{A}(s)-\int_{1}^{N} M_{1}^{\prime}(x) x^{-s} d x\right) \overline{\left(\mathbb{B}(s)-\int_{1}^{N} M_{2}^{\prime}(x) x^{-s} d x\right)} d t \\
= & \widehat{\Psi}_{U}(0) T \sum_{n \leq N} a_{n} \bar{b}_{n} n^{-2 \sigma}+T \sum_{1 \leq h \leq H} \int_{h \tau}^{N-h} M_{1}^{\prime}(x, h) K_{\sigma}(x, h) d x \\
& +T \sum_{1 \leq h \leq H} \int_{h \tau}^{N-h} \overline{M_{2}^{\prime}(x, h) K_{\sigma}(x, h)} d x \\
& -T \int_{\tau^{-1}}^{H} \int_{u \tau}^{N-u} M_{1}^{\prime}(x+u) \overline{M_{2}^{\prime}(x) K_{\sigma}(x, u)} d x d u \\
& -T \int_{\tau^{-1}}^{H} \int_{u \tau}^{N-u} \overline{M_{2}^{\prime}(x+u)} M_{1}^{\prime}(x) K_{\sigma}(x, u) d x d u \\
& +O\left(N^{1-2 \sigma+\max (\theta, \varphi)+5 \varepsilon}\right)+O\left(T^{2 \varepsilon}\right)
\end{aligned}
$$

uniformly for $\sigma_{1} \leq \sigma \leq \sigma_{2}$ and $T$ sufficiently large. 
The following weaker version of Theorem 1 is easier to apply and sufficient for many applications.

COROLlary 1. Let the hypotheses and notation be the same as in Theorem 1 except now assume that $N \gg T$ and that $\left(\mathrm{A}_{4}\right)$ also holds. Write

Then

$$
C_{T}=2 \pi\left(\frac{T}{2 \pi}\right)^{2-2 \sigma} .
$$

$$
\begin{aligned}
I= & \widehat{\Psi}_{U}(0) T \sum_{n \leq N} a_{n} \bar{b}_{n} n^{-2 \sigma} \\
& +C_{T} \int_{T /(2 \pi N)}^{\infty}\left(\sum_{1 \leq h \leq 2 \pi N v / T} M_{1}^{\prime}\left(\frac{h T}{2 \pi v}, h\right) h^{1-2 \sigma}\right) \widehat{\Psi}_{U}(v) v^{2 \sigma-2} d v \\
& +C_{T} \int_{T /(2 \pi N)}^{\infty}\left(\sum_{1 \leq h \leq 2 \pi N v / T} M_{2}^{\prime}\left(\frac{h T}{2 \pi v}, h\right) h^{1-2 \sigma}\right) \overline{\widehat{\Psi}_{U}(v)} v^{2 \sigma-2} d v \\
& -2 C_{T} \int_{T /(2 \pi \tau N)}^{\infty}\left(\int_{0}^{2 \pi N v / T} M_{1}^{\prime}\left(\frac{u T}{2 \pi v}\right) \overline{M_{2}^{\prime}\left(\frac{u T}{2 \pi v}\right)} u^{1-2 \sigma} d u\right) \\
& +O\left(T^{-1} N^{2-2 \sigma+5 \varepsilon}\right)+O\left(N^{1-2 \sigma+\max (\theta, \varphi)+5 \varepsilon}\right)+O\left(N^{2 \varepsilon}\right)
\end{aligned}
$$

uniformly for $\sigma_{1} \leq \sigma \leq \sigma_{2}$ and $T$ sufficiently large.

TheOREM 2. Suppose that the sequences $\left\{a_{n}\right\}_{n=1}^{\infty}$ and $\left\{b_{n}\right\}_{n=1}^{\infty}$ satisfy $\left(\mathrm{A}_{1}\right),\left(\mathrm{A}_{2}\right)$, and $\left(\mathrm{A}_{3}\right)$. Let $1<\sigma_{1}^{\prime}<\sigma_{2}^{\prime}$, let $0<\varepsilon<1 / 2$ be fixed, and set $\tau=T^{1-\varepsilon}$. For $\sigma_{1}^{\prime} \leq \sigma \leq \sigma_{2}^{\prime}$ write

let

$$
\lambda=\frac{2 \sigma-1}{2 \sigma-2}
$$

$$
\tau \ll N \leq \tau^{(1-\varepsilon)(1+\eta /(\lambda(1-\eta)))},
$$

where $\eta$ is as in $\left(\mathrm{A}_{3}\right)$, and set

$$
H^{*}=\tau^{-\lambda} N^{\lambda /(1-\varepsilon)} .
$$

Then

$$
\begin{aligned}
I^{*}= & \int_{-\infty}^{\infty} \Psi_{U}\left(\frac{t}{T}\right) \\
& \times\left(\mathbb{A}^{*}(s)-\int_{N}^{\infty} M_{1}^{\prime}(x) x^{-s} d x\right) \overline{\left(\mathbb{B}^{*}(s)-\int_{N}^{\infty} M_{2}^{\prime}(x) x^{-s} d x\right)} d t \\
= & \widehat{\Psi}_{U}(0) T \sum_{N<n} a_{n} \bar{b}_{n} n^{-2 \sigma}
\end{aligned}
$$




$$
\begin{aligned}
& +T \sum_{1 \leq h \leq H^{*}} \int_{\max (N, h \tau)}^{\infty} M_{1}^{\prime}(x, h) K_{\sigma}(x, h) d x \\
& +T \sum_{1 \leq h \leq H^{*}} \int_{\max (N, h \tau)}^{\infty} \overline{M_{2}^{\prime}(x, h) K_{\sigma}(x, h)} d x \\
& -T \int_{0}^{H^{*}} \int_{\max (N, u \tau)}^{\infty} M_{1}^{\prime}(x+u) \overline{M_{2}^{\prime}(x) K_{\sigma}(x, u)} d x d u \\
& -T \int_{0}^{H^{*}} \int_{\max (N, u \tau)}^{\infty} \overline{M_{2}^{\prime}(x+u)} M_{1}^{\prime}(x) K_{\sigma}(x, u) d x d u \\
& +O\left(N^{1-2 \sigma+\max (\theta, \varphi)+5 \varepsilon}\right)+O\left(T^{1-\varepsilon / 2} N^{1-2 \sigma}\right)
\end{aligned}
$$

uniformly for $\sigma_{1}^{\prime} \leq \sigma \leq \sigma_{2}^{\prime}$ and $T$ sufficiently large.

A simpler form of Theorem 2 is provided by

COROLlaRY 2. Let the hypotheses and notation be the same as in Theorem 2 except now assume that $N \gg T$ and that $\left(\mathrm{A}_{4}\right)$ also holds. Then

$$
\begin{aligned}
& I^{*}=\widehat{\Psi}_{U}(0) T \sum_{N<n} a_{n} \bar{b}_{n} n^{-2 \sigma} \\
& +C_{T} \int_{0}^{T /(2 \pi N)}\left(\sum_{1 \leq h \leq H^{*}} M_{1}^{\prime}\left(\frac{h T}{2 \pi v}, h\right) h^{1-2 \sigma}\right) \widehat{\Psi}_{U}(v) v^{2 \sigma-2} d v \\
& +C_{T} \int_{0}^{T /(2 \pi N)}\left(\sum_{1 \leq h \leq H^{*}} \overline{M_{2}^{\prime}\left(\frac{h T}{2 \pi v}, h\right)} h^{1-2 \sigma}\right) \overline{\widehat{\Psi}_{U}(v)} v^{2 \sigma-2} d v \\
& +C_{T} \int_{T /(2 \pi N)}^{T H^{*} /(2 \pi N)}\left(\sum_{2 \pi N v / T<h \leq H^{*}} M_{1}^{\prime}\left(\frac{h T}{2 \pi v}, h\right) h^{1-2 \sigma}\right) \widehat{\Psi}_{U}(v) v^{2 \sigma-2} d v \\
& +C_{T} \int_{T /(2 \pi N)}^{T H^{*} /(2 \pi N)}\left(\sum_{2 \pi N v / T<h \leq H^{*}} \overline{M_{2}^{\prime}\left(\frac{h T}{2 \pi v}, h\right)} h^{1-2 \sigma)} \overline{\widehat{\Psi}_{U}(v)} v^{2 \sigma-2} d v\right. \\
& -2 C_{T} \int_{0}^{T H^{*} /(2 \pi N)}\left(\int_{2 \pi N v / T}^{H^{*}} M_{1}^{\prime}\left(\frac{u T}{2 \pi v}\right) \overline{M_{2}^{\prime}\left(\frac{u T}{2 \pi v}\right)} u^{1-2 \sigma} d u\right) \\
& \times \operatorname{Re} \widehat{\Psi}_{U}(v) v^{2 \sigma-2} d v \\
& +O\left(T^{-1} N^{2-2 \sigma+5 \varepsilon}\right)+O\left(N^{1-2 \sigma+\max (\theta, \varphi)+5 \varepsilon}\right)+O\left(T^{1-\varepsilon / 2} N^{1-2 \sigma}\right)
\end{aligned}
$$

uniformly for $\sigma_{1}^{\prime} \leq \sigma \leq \sigma_{2}^{\prime}$ and $T$ sufficiently large, where $C_{T}$ is defined in Corollary 1. 
Although we could make the next theorem more precise by arguing along the lines of the proofs of Theorems 1 and 2, the version below is usually all that we require.

THEOREM 3. Assume that the sequences $\left\{a_{n}\right\}_{n=1}^{\infty}$ and $\left\{b_{n}\right\}_{n=1}^{\infty}$ satisfy $\left(\mathrm{A}_{1}\right)$ and (6) and that $N \gg T$. Let $\sigma_{1}<\sigma_{2}<1,1<\sigma_{1}^{\prime}<\sigma_{2}^{\prime}, s=\sigma+i$, $s^{\prime}=\sigma^{\prime}+$ it, and let $0<\varepsilon<1 / 2$ be arbitrary. Then

$$
\begin{aligned}
J & =\int_{-\infty}^{\infty} \Psi_{U}\left(\frac{t}{T}\right)\left(\mathbb{A}(s)-\int_{1}^{N} M_{1}^{\prime}(x) x^{-s} d x\right) \overline{\left(\mathbb{B}^{*}\left(s^{\prime}\right)-\int_{N}^{\infty} M_{2}^{\prime}(x) x^{-s^{\prime}} d x\right)} d t \\
& \ll T^{-1} N^{2-\sigma-\sigma^{\prime}+5 \varepsilon}
\end{aligned}
$$

uniformly for $\sigma_{1} \leq \sigma \leq \sigma_{2}$ and $\sigma_{1}^{\prime} \leq \sigma^{\prime} \leq \sigma_{2}^{\prime}$ and $T$ sufficiently large.

One measure of the strength of our results is how much larger than $T$ we may take $N$ and still retain an asymptotic formula. This is determined by the parameters $\theta, \varphi$, and $\eta$ as can be seen, for example, from (24) and the error term

$$
O\left(N^{1-2 \sigma+\max (\theta, \varphi)+5 \varepsilon}\right)
$$

in (26) of Theorem 1. It turns out that this term comes from using the pointwise upper bounds for $E_{i}(x)$ and $E_{i}(x, h)(i=1,2)$ given in $\left(\mathrm{A}_{2}\right)$ and $\left(\mathrm{A}_{3}\right)$ to estimate various expressions involving these functions. It is worth noting that if $E_{i}(x)$ and $E_{i}(x, h)(i=1,2)$ act like random variables in $x$ and behave independently as functions of $h$, then one might expect to be able to replace (32) by

$$
O\left(T^{1 / 2} N^{1 / 2-2 \sigma+\max (\theta, \varphi)+5 \varepsilon}\right) .
$$

This observation makes it easy to conjecture the mean values of very long Dirichlet polynomials as we shall illustrate in Example 3 of Section 5. We would similarly expect (33) to replace the next-to-last error term in Theorem 2 and Corollaries 1 and 2. It is also worth noting that one can sometimes exploit averages of $E_{i}(x, h)$ over $h$ to improve (32).

2. Proof of Theorem 1 and its corollary. Multiplying out in (11), we obtain

$$
\begin{aligned}
I= & \int_{-\infty}^{\infty} \Psi_{U}\left(\frac{t}{T}\right) \mathbb{A}(s) \overline{\mathbb{B}(s)} d t-\int_{-\infty}^{\infty} \Psi_{U}\left(\frac{t}{T}\right) \mathbb{A}(s) \overline{\left(\int_{1}^{N} M_{2}^{\prime}(x) x^{-s} d x\right)} d t \\
& -\int_{-\infty}^{\infty} \Psi_{U}\left(\frac{t}{T}\right) \overline{\mathbb{B}(s)}\left(\int_{1}^{N} M_{1}^{\prime}(x) x^{-s} d x\right) d t
\end{aligned}
$$




$$
+\int_{-\infty}^{\infty} \Psi_{U}\left(\frac{t}{T}\right)\left(\int_{1}^{N} M_{1}^{\prime}(x) x^{-s} d x\right) \overline{\left(\int_{1}^{N} M_{2}^{\prime}(y) y^{-s} d y\right)} d t
$$

or

$$
I=I_{1}-I_{2}-I_{3}+I_{4}
$$

First consider $I_{1}$. By (15) and (16) we have

$$
\begin{aligned}
I_{1}= & \widehat{\Psi}_{U}(0) T \sum_{n \leq N} a_{n} \bar{b}_{n} n^{-2 \sigma} \\
& +T \sum_{n<N} \sum_{n<m \leq N} a_{n} \bar{b}_{m}(m n)^{-\sigma} \widehat{\Psi}_{U}\left(\frac{T}{2 \pi} \log \frac{m}{n}\right) \\
& +T \sum_{n<N} \sum_{n<m \leq N} b_{n} \bar{a}_{m}(m n)^{-\sigma} \widehat{\Psi}_{U}\left(\frac{T}{2 \pi} \log \frac{m}{n}\right)
\end{aligned}
$$

or

$$
I_{1}=\widehat{\Psi}_{U}(0) T \sum_{n \leq N} a_{n} \bar{b}_{n} n^{-2 \sigma}+T I_{12}+T \bar{I}_{13}
$$

for short. In $I_{12}$ we set $m=n+h$ and note that by $\left(\mathrm{A}_{1}\right)$ and (19) the total contribution of those terms with $h>n / \tau$ is no more than $O\left(T^{-1}\right)$, say. It follows that

$$
\begin{aligned}
I_{12}= & \sum_{1 \leq n<N} \sum_{1 \leq h \leq \min (n / \tau, N-n)} a_{n} \bar{b}_{n+h} n^{-2 \sigma}\left(1+\frac{h}{n}\right)^{-\sigma} \widehat{\Psi}_{U}\left(\frac{T}{2 \pi} \log \left(1+\frac{h}{n}\right)\right) \\
& +O\left(T^{-1}\right) \\
= & \sum_{1 \leq n<N} \sum_{1 \leq h \leq \min (n / \tau, N-n)} a_{n} \bar{b}_{n+h} K_{\sigma}(n, h)+O\left(T^{-1}\right) .
\end{aligned}
$$

Changing the order of summation, we obtain

$$
I_{12}=\sum_{1 \leq h \leq H} \sum_{h \tau \leq n \leq N-h} a_{n} \bar{b}_{n+h} K_{\sigma}(n, h)+O\left(T^{-1}\right) .
$$

By (9) and Stieltjes integration this becomes

$$
\begin{aligned}
I_{12}= & \sum_{1 \leq h \leq H} \int_{h \tau}^{N-h} M_{1}^{\prime}(x, h) K_{\sigma}(x, h) d x \\
& +\sum_{1 \leq h \leq H} \int_{h \tau^{-}}^{N-h} K_{\sigma}(x, h) d E_{1}(x, h)+O\left(T^{-1}\right) .
\end{aligned}
$$


The second term equals

$$
\sum_{1 \leq h \leq H}\left(\left.E_{1}(x, h) K_{\sigma}(x, h)\right|_{h \tau^{-}} ^{N-h}-\int_{h \tau}^{N-h} E_{1}(x, h) \frac{\partial}{\partial x} K_{\sigma}(x, h) d x\right) .
$$

To bound this we use (10), but first we must check that $h \leq x^{\eta}$ whenever $1 \leq h \leq H$ and $x \in[h \tau, N-h]$. This will be the case if $h \leq(h \tau)^{\eta}$ for every $h \leq H$, or if

$$
H \leq \tau^{\eta /(1-\eta)} .
$$

But this follows from (24) and (25). By (10), (20), and (21) we now find that (36) is

$$
\ll T^{\varepsilon} \sum_{1 \leq h \leq H}\left((h \tau)^{\varphi-2 \sigma+\varepsilon / 2}+N^{\varphi-2 \sigma+\varepsilon / 2}\right) .
$$

Here we have appealed to the estimate

$$
\int_{A}^{B} x^{\lambda} d x \ll A^{1+\lambda+\delta}+B^{1+\lambda+\delta},
$$

which holds uniformly for $1 \leq A \leq B$ and bounded $\lambda$, where $\delta>0$ is arbitrarily small, and where the implied constant depends at most on $\delta$. We also note for later use that the $\delta$ is unnecessary if $\lambda$ is bounded away from -1 . Next, using the discrete analogue of this, namely

$$
\sum_{A \leq h \leq B} h^{\lambda} \ll A^{1+\lambda+\delta}+B^{1+\lambda+\delta},
$$

we see that the sum above is

$$
\begin{aligned}
& \ll T^{\varepsilon} \tau^{\varphi-2 \sigma+\varepsilon}\left(H^{1+\varphi-2 \sigma+\varepsilon}+1\right)+T^{\varepsilon} H N^{\varphi-2 \sigma+\varepsilon} \\
& \ll T^{\varepsilon} \tau^{-1}\left(N^{1+\varphi-2 \sigma+\varepsilon}+\tau^{1+\varphi-2 \sigma+\varepsilon}\right) \\
& \ll T^{-1}\left(N^{1+\varphi-2 \sigma+5 \varepsilon}+\tau^{1+\varphi-2 \sigma+5 \varepsilon}\right),
\end{aligned}
$$

since

$$
\tau^{\varepsilon}<T^{\varepsilon}<\tau^{2 \varepsilon}<N^{2 \varepsilon}
$$

when $0<\varepsilon<1 / 2$. If $1+\varphi-2 \sigma+5 \varepsilon \leq 0$, this is $\ll T^{-1}$ and in the opposite case it is $\ll T^{-1} N^{1+\varphi-2 \sigma+5 \varepsilon}$, because $\tau \ll N$. Thus, (36) is $\ll T^{-1} N^{1+\varphi-2 \sigma+5 \varepsilon}+T^{-1}$ and it follows that

$$
I_{12}=\sum_{1 \leq h \leq H} \int_{h \tau}^{N-h} M_{1}^{\prime}(x, h) K_{\sigma}(x, h) d x+O\left(T^{-1} N^{1+\varphi-2 \sigma+5 \varepsilon}\right)+O\left(T^{-1}\right) .
$$

Treating $I_{13}$ in the same way, we obtain

$$
I_{13}=\sum_{1 \leq h \leq H} \int_{h \tau}^{N-h} M_{2}^{\prime}(x, h) K_{\sigma}(x, h) d x+O\left(T^{-1} N^{1+\varphi-2 \sigma+5 \varepsilon}\right)+O\left(T^{-1}\right) .
$$


Combining these results with (35), we now find that

$$
\begin{aligned}
& I_{1}=\widehat{\Psi}_{U}(0) T \sum_{n \leq N} a_{n} \bar{b}_{n} n^{-2 \sigma} \\
& +T \sum_{1 \leq h \leq H} \int_{h \tau}^{N-h} M_{1}^{\prime}(x, h) K_{\sigma}(x, h) d x \\
& +T \sum_{1 \leq h \leq H} \int_{h \tau}^{N-h} \overline{M_{2}^{\prime}(x, h) K_{\sigma}(x, h)} d x+O\left(N^{1+\varphi-2 \sigma+5 \varepsilon}\right)+O(1) .
\end{aligned}
$$

Next we treat $I_{2}$. By (15) we have

$$
\begin{aligned}
I_{2} & =T \sum_{n \leq N} a_{n} n^{-\sigma} \int_{1}^{N} \overline{M_{2}^{\prime}(x)} x^{-\sigma} \widehat{\Psi}_{U}\left(\frac{T}{2 \pi} \log \frac{x}{n}\right) d x \\
& =T \sum_{n \leq N} a_{n} n^{-\sigma}\left(\int_{n}^{N}+\int_{1}^{n}\right) \overline{M_{2}^{\prime}(x)} x^{-\sigma} \widehat{\Psi}_{U}\left(\frac{T}{2 \pi} \log \frac{x}{n}\right) d x
\end{aligned}
$$

or

$$
I_{2}=T I_{21}+T I_{22}
$$

In $I_{21}$ we set $x=n+u$ and note as before that by $\left(\mathrm{A}_{1}\right),(6)$, and (19), that portion of the integral with $u>n / \tau$ contributes a negligible amount. Thus we find that

$$
\begin{aligned}
I_{21}= & \sum_{n \leq N} a_{n} n^{-\sigma} \int_{0}^{\min (n / \tau, N-n)} \overline{M_{2}^{\prime}(n+u)}(n+u)^{-\sigma} \widehat{\Psi}_{U}\left(\frac{T}{2 \pi} \log \left(1+\frac{u}{n}\right)\right) d u \\
& +O\left(T^{-1}\right) \\
= & \sum_{n \leq N} a_{n} \int_{0}^{\min (n / \tau, N-n)} \overline{M_{2}^{\prime}(n+u)} K_{\sigma}(n, u) d u+O\left(T^{-1}\right),
\end{aligned}
$$

say. Changing the order of summation and integration, we find that

$$
\begin{aligned}
I_{21}= & \int_{0}^{\tau^{-1}} \sum_{n \leq N-u} a_{n} \overline{M_{2}^{\prime}(n+u)} K_{\sigma}(n, u) d u \\
& +\int_{\tau^{-1}}^{H} \sum_{u \tau \leq n \leq N-u} a_{n} \overline{M_{2}^{\prime}(n+u)} K_{\sigma}(n, u) d u+O\left(T^{-1}\right) .
\end{aligned}
$$

The first term is

$$
\ll \int_{0}^{\tau^{-1}}\left(\sum_{n \leq N} n^{\varepsilon-2 \sigma}\right) d u \ll \tau^{-1}\left(N^{1-2 \sigma+2 \varepsilon}+1\right) \ll T^{-1}\left(N^{1-2 \sigma+4 \varepsilon}+T^{2 \varepsilon}\right)
$$


by $\left(\mathrm{A}_{1}\right),(6),(20)$ and $(37 \mathrm{a})$. By (4) and Stieltjes integration the second term equals

$$
\begin{aligned}
\int_{\tau^{-1}}^{H} \int_{u \tau}^{N-u} M_{1}^{\prime}(x) \overline{M_{2}^{\prime}(x+u)} & K_{\sigma}(x, u) d x d u \\
& +\int_{\tau^{-1}}^{H} \int_{u \tau}^{N-u} \overline{M_{2}^{\prime}(x+u)} K_{\sigma}(x, u) d E_{1}(x) d u .
\end{aligned}
$$

Integrating by parts and using (6)-(8), (20), (21), and (37b), we see that the second term is

$$
\begin{aligned}
& =\int_{\tau^{-1}}^{H}\left(\left.\overline{M_{2}^{\prime}(x+u)} K_{\sigma}(x, u) E_{1}(x)\right|_{u \tau} ^{N-u}\right) d u \\
& \quad-\int_{\tau^{-1}}^{H} \int_{u \tau}^{N-u}\left(\overline{M_{2}^{\prime \prime}(x+u)} K_{\sigma}(x, u)+\overline{M_{2}^{\prime}(x+u)} \frac{\partial}{\partial x} K_{\sigma}(x, u)\right) E_{1}(x) d x d u \\
& \ll T^{\varepsilon} \int_{\tau^{-1}}^{H}\left((u \tau)^{\theta-2 \sigma+\varepsilon / 2}+N^{\theta-2 \sigma+\varepsilon / 2}\right) d u \\
& \ll T^{\varepsilon} \tau^{-1} \int_{1}^{N} v^{\theta-2 \sigma+\varepsilon / 2} d v+T^{\varepsilon} H N^{\theta-2 \sigma+\varepsilon / 2} \\
& \ll T^{\varepsilon} \tau^{-1}\left(N^{1+\theta-2 \sigma+\varepsilon}+1\right) \ll T^{-1}\left(N^{1+\theta-2 \sigma+5 \varepsilon}+T^{2 \varepsilon}\right) .
\end{aligned}
$$

Thus we have

$$
\begin{aligned}
I_{21}= & \int_{\tau^{-1}}^{H} \int_{u \tau}^{N-u} M_{1}^{\prime}(x) \overline{M_{2}^{\prime}(x+u)} K_{\sigma}(x, u) d x d u \\
& +O\left(T^{-1} N^{1+\theta-2 \sigma+5 \varepsilon}\right)+O\left(T^{-1+2 \varepsilon}\right) .
\end{aligned}
$$

We treat $I_{22}$ similarly. Setting $x=n-u$, we see that

$$
I_{22}=\sum_{n \leq N} a_{n} n^{-\sigma} \int_{0}^{n-1} \overline{M_{2}^{\prime}(n-u)}(n-u)^{-\sigma} \widehat{\Psi}_{U}\left(\frac{T}{2 \pi} \log \left(1-\frac{u}{n}\right)\right) d u .
$$

Using $\left(\mathrm{A}_{1}\right),(6)$, and (19) for that part of the integral for which $u>n /(\tau+1)$, we find that

$$
\begin{gathered}
\sum_{n \leq N} a_{n} n^{-\sigma} \int_{0}^{\min (n /(\tau+1), n-1)} \overline{M_{2}^{\prime}(n-u)}(n-u)^{-\sigma} \widehat{\Psi}_{U}\left(\frac{T}{2 \pi} \log \left(1-\frac{u}{n}\right)\right) d u \\
+O\left(T^{-1}\right)=\sum_{2 \leq n \leq N} a_{n} \int_{0}^{n /(\tau+1)} \overline{M_{2}^{\prime}(n-u) K_{\sigma}(n-u, u)} d u+O\left(T^{-1}\right) .
\end{gathered}
$$


If we change the order of summation and integration we obtain

$$
\begin{aligned}
I_{22}= & \int_{0}^{\tau^{-1}} \sum_{2 \leq n \leq N} a_{n} \overline{M_{2}^{\prime}(n-u) K_{\sigma}(n-u, u)} d u \\
& +\int_{\tau^{-1}}^{H} \sum_{u(\tau+1) \leq n \leq N} a_{n} \overline{M_{2}^{\prime}(n-u) K_{\sigma}(n-u, u)} d u+O\left(T^{-1}\right) .
\end{aligned}
$$

As in the case of $I_{21}$, the first term is easily seen to be $\ll T^{-1}\left(N^{1-2 \sigma+4 \varepsilon}+\right.$ $\left.T^{2 \varepsilon}\right)$. Hence we have

$$
\begin{aligned}
I_{22}= & \int_{\tau^{-1}}^{H} \sum_{u(\tau+1) \leq n \leq N} a_{n} \overline{M_{2}^{\prime}(n-u) K_{\sigma}(n-u, u)} d u \\
& +O\left(T^{-1} N^{1-2 \sigma+4 \varepsilon}\right)+O\left(T^{-1+2 \varepsilon}\right) .
\end{aligned}
$$

By (4) and Stieltjes integration we may write this as

$$
\begin{aligned}
I_{22}= & \int_{\tau^{-1}}^{H} \int_{u(\tau+1)}^{N} M_{1}^{\prime}(y) \overline{M_{2}^{\prime}(y-u) K_{\sigma}(y-u, u)} d y d u \\
& +\int_{\tau^{-1}}^{H} \int_{u(\tau+1)}^{N} \overline{M_{2}^{\prime}(y-u) K_{\sigma}(y-u, u)} d E_{1}(y) d u \\
& +O\left(T^{-1} N^{1-2 \sigma+4 \varepsilon}\right)+O\left(T^{-1+2 \varepsilon}\right) .
\end{aligned}
$$

If we estimate the second term as was done for the corresponding term in (40), we see that it also is $\ll T^{-1}\left(N^{1+\theta-2 \sigma+5 \varepsilon}+T^{2 \varepsilon}\right)$. In the first term we replace $y$ by $x+u$. We then obtain

$$
\begin{aligned}
I_{22}= & \int_{\tau^{-1}}^{H} \int_{u \tau}^{N-u} M_{1}^{\prime}(x+u) \overline{M_{2}^{\prime}(x) K_{\sigma}(x, u)} d x d u \\
& +O\left(T^{-1} N^{1+\theta-2 \sigma+5 \varepsilon}\right)+O\left(T^{-1+2 \varepsilon}\right) .
\end{aligned}
$$

Combining this with (41) in (39) we now find that

$$
\begin{aligned}
I_{2}= & T \int_{\tau^{-1}}^{H} \int_{u \tau}^{N-u} M_{1}^{\prime}(x+u) \overline{M_{2}^{\prime}(x) K_{\sigma}(x, u)} d x d u \\
& +T \int_{\tau^{-1}}^{H} \int_{u \tau}^{N-u} \overline{M_{2}^{\prime}(x+u)} M_{1}^{\prime}(x) K_{\sigma}(x, u) d x d u \\
& +O\left(N^{1+\theta-2 \sigma+5 \varepsilon}\right)+O\left(T^{2 \varepsilon}\right) .
\end{aligned}
$$

Clearly $I_{3}$ is the complex conjugate of $I_{2}$, but with $\mathbb{B}(s)$ instead of $\mathbb{A}(s)$ 
and $M_{1}^{\prime}(x)$ instead of $M_{2}^{\prime}(x)$. It therefore follows from (42) that

$$
\begin{aligned}
I_{3}= & T \int_{\tau^{-1}}^{H} \int_{u \tau}^{N-u} \overline{M_{2}^{\prime}(x+u)} M_{1}^{\prime}(x) K_{\sigma}(x, u) d x d u \\
& +T \int_{\tau^{-1}}^{H} \int_{u \tau}^{N-u} M_{1}^{\prime}(x+u) \overline{M_{2}^{\prime}(x) K_{\sigma}(x, u)} d x d u \\
& +O\left(N^{1+\theta-2 \sigma+5 \varepsilon}\right)+O\left(T^{2 \varepsilon}\right),
\end{aligned}
$$

which is identical to the expression for $I_{2}$.

Finally, we come to $I_{4}$. By (15) and (16) we see that

$$
\begin{aligned}
I_{4}= & T \int_{1}^{N} \int_{x}^{N} M_{1}^{\prime}(x) \overline{M_{2}^{\prime}(y)}(x y)^{-\sigma} \widehat{\Psi}_{U}\left(\frac{T}{2 \pi} \log \frac{y}{x}\right) d y d x \\
& +T \int_{1}^{N} \int_{x}^{N} M_{2}^{\prime}(x) \overline{M_{1}^{\prime}(y)}(x y)^{-\sigma} \widehat{\Psi}_{U}\left(\frac{T}{2 \pi} \log \frac{y}{x}\right) d y d x \\
= & T I_{41}+T \bar{I}_{42},
\end{aligned}
$$

say. In $I_{41}$ we set $y=x+u$ and use (6) and (19) for $u>x / \tau$ to obtain

$$
\begin{aligned}
I_{41}= & \int_{1}^{N} \int_{0}^{\min (x / \tau, N-x)} M_{1}^{\prime}(x) \overline{M_{2}^{\prime}(x+u)} x^{-2 \sigma}\left(1+\frac{u}{x}\right)^{-\sigma} \\
& \times \widehat{\Psi}_{U}\left(\frac{T}{2 \pi} \log \left(1+\frac{u}{x}\right)\right) d u d x+O\left(T^{-1}\right) \\
= & \int_{1}^{N} \int_{0}^{\min (x / \tau, N-x)} M_{1}^{\prime}(x) \overline{M_{2}^{\prime}(x+u)} K_{\sigma}(x, u) d u d x+O\left(T^{-1}\right) .
\end{aligned}
$$

Next we change the order of integration and find that

$$
\begin{aligned}
I_{41}= & \int_{0}^{\tau^{-1}} \int_{1}^{N-u} M_{1}^{\prime}(x) \overline{M_{2}^{\prime}(x+u)} K_{\sigma}(x, u) d x d u \\
& +\int_{\tau^{-1}}^{H} \int_{u \tau}^{N-u} M_{1}^{\prime}(x) \overline{M_{2}^{\prime}(x+u)} K_{\sigma}(x, u) d x d u+O\left(T^{-1}\right) .
\end{aligned}
$$

By (6), (20), and (37a) the first term is

$$
\ll \tau^{-1} \int_{1}^{N} x^{\varepsilon-2 \sigma} d x \ll T^{-1+\varepsilon}\left(N^{1-2 \sigma+2 \varepsilon}+1\right) \ll T^{-1}\left(N^{1-2 \sigma+4 \varepsilon}+T^{\varepsilon}\right) .
$$


Thus,

$$
\begin{aligned}
I_{41}= & \int_{\tau^{-1}}^{H} \int_{u \tau}^{N-u} M_{1}^{\prime}(x) \overline{M_{2}^{\prime}(x+u)} K_{\sigma}(x, u) d x d u \\
& +O\left(T^{-1} N^{1-2 \sigma+4 \varepsilon}\right)+O\left(T^{-1+\varepsilon}\right) .
\end{aligned}
$$

Since $I_{42}$ is $I_{41}$ with $M_{1}^{\prime}$ and $M_{2}^{\prime}$ interchanged, we also have

$$
\begin{aligned}
I_{42}= & \int_{\tau^{-1}}^{H} \int_{u \tau}^{N-u} M_{2}^{\prime}(x) \overline{M_{1}^{\prime}(x+u)} K_{\sigma}(x, u) d x d u \\
& +O\left(T^{-1} N^{1-2 \sigma+4 \varepsilon}\right)+O\left(T^{-1+\varepsilon}\right) .
\end{aligned}
$$

Thus we find that

$$
\begin{aligned}
I_{4}= & T \int_{\tau^{-1}}^{H} \int_{u \tau}^{N-u} M_{1}^{\prime}(x+u) \overline{M_{2}^{\prime}(x) K_{\sigma}(x, u)} d x d u \\
& +T \int_{\tau^{-1}}^{H} \int_{u \tau}^{N-u} \overline{M_{2}^{\prime}(x+u)} M_{1}^{\prime}(x) K_{\sigma}(x, u) d x d u \\
& +O\left(N^{1-2 \sigma+4 \varepsilon}\right)+O\left(T^{\varepsilon}\right) .
\end{aligned}
$$

On combining (34), (38), and (42), (43), and (45), we obtain

$$
\begin{aligned}
I= & \widehat{\Psi}_{U}(0) T \sum_{n \leq N} a_{n} \bar{b}_{n} n^{-2 \sigma} \\
& +T \sum_{1 \leq h \leq H} \int_{h \tau}^{N-h} M_{1}^{\prime}(x, h) K_{\sigma}(x, h) d x \\
& +T \sum_{1 \leq h \leq H} \int_{h \tau}^{N-h} \overline{M_{2}^{\prime}(x, h) K_{\sigma}(x, h)} d x \\
& -T \int_{\tau^{-1}}^{H} \int_{u \tau}^{N-u} M_{1}^{\prime}(x+u) \overline{M_{2}^{\prime}(x) K_{\sigma}(x, u)} d x d u \\
& -T \int_{\tau^{-1}}^{H} \int_{u \tau}^{N-u} \overline{M_{2}^{\prime}(x+u)} M_{1}^{\prime}(x) K_{\sigma}(x, u) d x d u \\
& +O\left(N^{1-2 \sigma+\max (\theta, \varphi)+5 \varepsilon}\right)+O\left(T^{2 \varepsilon}\right) .
\end{aligned}
$$

This agrees with (26) so the proof of Theorem 1 is complete.

We now deduce Corollary 1 from Theorem 1 . In the second term on the right in (46) we replace $N-h$ by $N$ and $H$ by $N / \tau$. Then by $\left(\mathrm{A}_{4}\right)$ and (20) 
this results in a change of at most

$$
\begin{aligned}
& \ll T \sum_{1 \leq h \leq H} h(h N)^{\varepsilon / 2}\left((h \tau)^{-2 \sigma}+N^{-2 \sigma}\right) \\
& \quad+T \sum_{N /(\tau+1)<h \leq N / \tau} h^{\varepsilon / 2} \int_{N \tau /(\tau+1)}^{N} x^{-2 \sigma+\varepsilon / 2} d x \\
& \ll T N^{\varepsilon / 2}\left(\tau^{-2 \sigma}\left(H^{2-2 \sigma+\varepsilon}+1\right)+N^{-2 \sigma}\left(H^{2+\varepsilon}+1\right)\right)+T \tau^{-3} N^{2-2 \sigma+\varepsilon} \\
& \ll T N^{\varepsilon / 2}\left(\tau^{-2 \sigma}(N / \tau)^{2-2 \sigma+\varepsilon}+N^{-2 \sigma}(N / \tau)^{2+\varepsilon}\right)+T \tau^{-3} N^{2-2 \sigma+\varepsilon} \\
& \ll T \tau^{-2} N^{2-2 \sigma+2 \varepsilon} \ll T^{-1} N^{2-2 \sigma+4 \varepsilon}
\end{aligned}
$$

since $\sigma<1$ and $T \ll N$. Hence the second term on the right-hand side of (46) equals

$$
T \sum_{1 \leq h \leq N / \tau} \int_{h \tau}^{N} M_{1}^{\prime}(x, h) K_{\sigma}(x, h) d x+O\left(T^{-1} N^{2-2 \sigma+4 \varepsilon}\right) .
$$

By (23) we may replace $K_{\sigma}(x, h)$ by $x^{-2 \sigma} \widehat{\Psi}_{U}\left(\frac{h T}{2 \pi x}\right)$ with a total error of at most

$$
\begin{aligned}
& \ll T^{2 \varepsilon} N^{\varepsilon / 4} \sum_{1 \leq h \leq N / \tau} \int_{h \tau}^{N} x^{-2 \sigma+\varepsilon / 4} d x \\
& \ll T^{2 \varepsilon} N^{\varepsilon / 4} \sum_{1 \leq h \leq N / \tau}\left((h \tau)^{1-2 \sigma+\varepsilon / 2}+N^{1-2 \sigma+\varepsilon / 2}\right) \\
& \ll T^{2 \varepsilon} N^{\varepsilon / 4}\left(\tau^{1-2 \sigma+\varepsilon / 2}\left((N / \tau)^{2-2 \sigma+\varepsilon}+1\right)+\tau^{-1} N^{2-2 \sigma+\varepsilon / 2}\right) \\
& \ll T^{-1+3 \varepsilon} N^{\varepsilon / 4}\left(N^{2-2 \sigma+\varepsilon}+\tau^{2-2 \sigma+\varepsilon}\right) \ll T^{-1} N^{2-2 \sigma+5 \varepsilon} .
\end{aligned}
$$

Thus, the expression above equals

$$
T \sum_{1 \leq h \leq N / \tau} \int_{h \tau}^{N} M_{1}^{\prime}(x, h) x^{-2 \sigma} \widehat{\Psi}_{U}\left(\frac{h T}{2 \pi x}\right) d x+O\left(T^{-1} N^{2-2 \sigma+5 \varepsilon}\right) .
$$

If we write $v$ for $h T /(2 \pi x)$ and then change the order of summation and integration, we get

$$
\begin{aligned}
C_{T} \int_{T /(2 \pi N)}^{T /(2 \pi \tau)}\left(\sum_{1 \leq h \leq 2 \pi N v / T} M_{1}^{\prime}\left(\frac{h T}{2 \pi v}, h\right) h^{1-2 \sigma}\right) \widehat{\Psi}_{U}(v) v^{2 \sigma-2} d v \\
+O\left(T^{-1} N^{2-2 \sigma+5 \varepsilon}\right) .
\end{aligned}
$$

Finally, by $\left(\mathrm{A}_{4}\right),(18)$, and (19) if we extend the interval of integration to infinity we change our term by a negligible amount. Thus, the second term 
on the right-hand side of (46) is

$$
\begin{aligned}
C_{T} \int_{T /(2 \pi N)}^{\infty}\left(\sum_{1 \leq h \leq 2 \pi N v / T} M_{1}^{\prime}\left(\frac{h T}{2 \pi v}, h\right) h^{1-2 \sigma}\right) \widehat{\Psi}_{U}(v) v^{2 \sigma-2} d v \\
+O\left(T^{-1} N^{2-2 \sigma+5 \varepsilon}\right) .
\end{aligned}
$$

Similarly, we see that the third term on the right-hand side of (46) is

$$
\begin{aligned}
C_{T} \int_{T /(2 \pi N)}^{\infty}\left(\sum_{1 \leq h \leq 2 \pi N v / T} \overline{M_{2}^{\prime}\left(\frac{h T}{2 \pi v}, h\right)} h^{1-2 \sigma}\right) \overline{\widehat{\Psi}_{U}(v)} v^{2 \sigma-2} d v \\
+O\left(T^{-1} N^{2-2 \sigma+5 \varepsilon}\right) .
\end{aligned}
$$

In much the same way we find that the fourth term on the right in (46) equals

$$
\begin{aligned}
-T \int_{\tau^{-1}}^{N / \tau} \int_{u \tau}^{N} M_{1}^{\prime}(x+u) \overline{M_{2}^{\prime}(x)} x^{-2 \sigma} \overline{\widehat{\Psi}_{U}\left(\frac{u T}{2 \pi x}\right)} & d x d u \\
& +O\left(T^{-1} N^{2-2 \sigma+5 \varepsilon}\right) .
\end{aligned}
$$

Now by (7) and the mean value theorem of differential calculus we have

$$
M_{1}^{\prime}(x+u)=M_{1}^{\prime}(x)+O\left((x+1)^{\varepsilon / 2} \tau^{-1}\right)
$$

for $u / x \leq \tau^{-1}$. Hence, replacing $M_{1}^{\prime}(x+u)$ by $M_{1}^{\prime}(x)$ and using the estimates $\sigma<1$ and $T \ll N$, and (17) we change the above by at most

$$
\begin{aligned}
& \ll T \tau^{-1} \int_{\tau^{-1}}^{N / \tau}\left(\int_{u \tau}^{N} x^{\varepsilon-2 \sigma} d x\right) d u \\
& \ll T \tau^{-1} \int_{\tau^{-1}}^{N / \tau}\left((u \tau)^{1-2 \sigma+2 \varepsilon}+N^{1-2 \sigma+2 \varepsilon}\right) d u \\
& \ll T \tau^{-1}\left(\tau^{-1} \int_{1}^{N} y^{1-2 \sigma+2 \varepsilon} d y+\tau^{-1} N^{2-2 \sigma+2 \varepsilon}\right) \\
& \ll T^{-1+2 \varepsilon}\left(N^{2-2 \sigma+3 \varepsilon}+1\right) \ll T^{-1} N^{2-2 \sigma+5 \varepsilon} .
\end{aligned}
$$

Thus, (47) equals

$$
-T \int_{\tau^{-1}}^{N / \tau} \int_{u \tau}^{N} M_{1}^{\prime}(x) \overline{M_{2}^{\prime}(x)} x^{-2 \sigma} \overline{\widehat{\Psi}_{U}\left(\frac{u T}{2 \pi x}\right)} d x d u+O\left(T^{-1} N^{2-2 \sigma+5 \varepsilon}\right) .
$$

Substituting $v$ for $u T /(2 \pi x)$ and then changing the order of integration, we 
find that this equals

$$
\begin{array}{r}
-C_{T} \int_{T /(2 \pi \tau N)}^{T /(2 \pi \tau)}\left(\int_{\tau^{-1}}^{2 \pi N v / T} M_{1}^{\prime}\left(\frac{u T}{2 \pi v}\right) \overline{M_{2}^{\prime}\left(\frac{u T}{2 \pi v}\right)} u^{1-2 \sigma} d u\right) \\
+O\left(T^{-1} N^{2-2 \sigma+5 \varepsilon}\right) .
\end{array}
$$

Now by $(6), M_{i}^{\prime}\left(\frac{u T}{2 \pi v}\right) \ll N^{\varepsilon}(i=1,2)$ in the rectangle $\left[0, \tau^{-1}\right] \times[T /(2 \pi N \tau)$, $T /(2 \pi \tau)]$. Using this and (17), we find that if we begin the $u$ integral at zero, the first term changes by

$$
\begin{aligned}
& \ll N^{\varepsilon}(T / \tau)^{2-2 \sigma} \int_{T /(2 \pi N \tau)}^{T /(2 \pi \tau)}\left|\widehat{\Psi}_{U}(v)\right| v^{2 \sigma-2} d v \\
& \ll N^{\varepsilon}(T / \tau)^{2-2 \sigma}\left((T / \tau)^{2 \sigma-1}+(T /(N \tau))^{2 \sigma-1}\right) \\
& \ll N^{\varepsilon} T \tau^{-1}\left(1+N^{1-2 \sigma}\right) \ll N^{2 \varepsilon}+N^{1-2 \sigma+2 \varepsilon} .
\end{aligned}
$$

Moreover, if we then extend the $v$ integral to infinity, this changes our expression by a negligible amount because of (6) and (18). Thus, (47) equals

$$
\begin{array}{r}
-C_{T} \int_{T /(2 \pi \tau N)}^{\infty}\left(\int_{0}^{2 \pi N v / T} M_{1}^{\prime}\left(\frac{u T}{2 \pi v}\right) \overline{M_{2}^{\prime}\left(\frac{u T}{2 \pi v}\right)} u^{1-2 \sigma} d u\right) \widehat{\widehat{\Psi}_{U}(v)} v^{2 \sigma-2} d v \\
+O\left(T^{-1} N^{2-2 \sigma+5 \varepsilon}\right)+O\left(N^{2 \varepsilon}\right) .
\end{array}
$$

Treating the fifth term in (46) in exactly the same way, we find that it equals

$$
\begin{array}{r}
-C_{T} \int_{T /(2 \pi \tau N)}^{\infty}\left(\int_{0}^{2 \pi N v / T} M_{1}^{\prime}\left(\frac{u T}{2 \pi v}\right) \overline{M_{2}^{\prime}\left(\frac{u T}{2 \pi v}\right)} u^{1-2 \sigma} d u\right) \widehat{\Psi}_{U}(v) v^{2 \sigma-2} d v \\
+O\left(T^{-1} N^{2-2 \sigma+5 \varepsilon}\right)+O\left(N^{2 \varepsilon}\right) .
\end{array}
$$

Combining all our results, we now obtain

$$
\begin{aligned}
I= & \widehat{\Psi}_{U}(0) T \sum_{n \leq N} a_{n} \bar{b}_{n} n^{-2 \sigma} \\
& +C_{T} \int_{T /(2 \pi N)}^{\infty}\left(\sum_{1 \leq h \leq 2 \pi N v / T} M_{1}^{\prime}\left(\frac{h T}{2 \pi v}, h\right) h^{1-2 \sigma}\right) \widehat{\Psi}_{U}(v) v^{2 \sigma-2} d v \\
& +C_{T} \int_{T /(2 \pi N)}^{\infty}\left(\sum_{1 \leq h \leq 2 \pi N v / T} M_{2}^{\prime}\left(\frac{h T}{2 \pi v}, h\right) h^{1-2 \sigma}\right) \overline{\widehat{\Psi}_{U}(v)} v^{2 \sigma-2} d v \\
& -2 C_{T} \int_{T /(2 \pi \tau N)}^{\infty}\left(\int_{0}^{2 \pi N v / T} M_{1}^{\prime}\left(\frac{u T}{2 \pi v}\right) \overline{M_{2}^{\prime}\left(\frac{u T}{2 \pi v}\right)} u^{1-2 \sigma} d u\right) \\
& \times \operatorname{Re} \widehat{\Psi}_{U}(v) v^{2 \sigma-2} d v
\end{aligned}
$$




$$
+O\left(T^{-1} N^{2-2 \sigma+5 \varepsilon}\right)+O\left(N^{1-2 \sigma+\max (\theta, \varphi)+5 \varepsilon}\right)+O\left(N^{2 \varepsilon}\right),
$$

which is the same as (27). Thus, the proof of Corollary 1 is complete.

3. Proof of Theorem 2 and its corollary. Multiplying out in (12), we have

$$
\begin{aligned}
I^{*}= & \int_{-\infty}^{\infty} \Psi_{U}\left(\frac{t}{T}\right) \mathbb{A}^{*}(s) \overline{\mathbb{B}^{*}(s)} d t-\int_{-\infty}^{\infty} \Psi_{U}\left(\frac{t}{T}\right) \mathbb{A}^{*}(s) \overline{\left(\int_{N}^{\infty} M_{2}^{\prime}(x) x^{-s} d x\right)} d t \\
& -\int_{-\infty}^{\infty} \Psi_{U}\left(\frac{t}{T}\right) \overline{\mathbb{B}^{*}(s)}\left(\int_{N}^{\infty} M_{1}^{\prime}(x) x^{-s} d x\right) d t \\
& +\int_{-\infty}^{\infty} \Psi_{U}\left(\frac{t}{T}\right)\left(\int_{N}^{\infty} M_{1}^{\prime}(x) x^{-s} d x\right) \overline{\left(\int_{N}^{\infty} M_{2}^{\prime}(y) y^{-s} d y\right)} d t
\end{aligned}
$$

or

$$
I^{*}=I_{1}^{*}-I_{2}^{*}-I_{3}^{*}+I_{4}^{*} .
$$

In $I_{1}^{*}$ we multiply the two series and note by $\left(\mathrm{A}_{1}\right)$ and our assumption that $\sigma>1$ that the resulting double series is absolutely convergent. We may therefore integrate termwise. Using (15) and (16), we then find that

$$
\begin{aligned}
I_{1}^{*}= & \widehat{\Psi}_{U}(0) T \sum_{N<n} a_{n} \bar{b}_{n} n^{-2 \sigma} \\
& +T \sum_{N<n} \sum_{n<m} a_{n} \bar{b}_{m}(m n)^{-\sigma} \widehat{\Psi}_{U}\left(\frac{T}{2 \pi} \log \frac{m}{n}\right) \\
& +T \sum_{N<n} \sum_{n<m} b_{n} \bar{a}_{m}(m n)^{-\sigma} \widehat{\Psi}_{U}\left(\frac{T}{2 \pi} \log \frac{m}{n}\right),
\end{aligned}
$$

or

$$
I_{1}^{*}=\widehat{\Psi}_{U}(0) T \sum_{N<n} a_{n} \bar{b}_{n} n^{-2 \sigma}+T I_{12}^{*}+T \overline{I_{13}^{*}} .
$$

Setting $m=n+h$ in $I_{12}^{*}$ and using $\left(\mathrm{A}_{1}\right)$ and (19) for $h \geq n / \tau$, we see that

$$
\begin{aligned}
I_{12}^{*}= & \sum_{N<n} \sum_{1 \leq h<n / \tau} a_{n} \bar{b}_{n+h} n^{-2 \sigma}\left(1+\frac{h}{n}\right)^{-\sigma} \widehat{\Psi}_{U}\left(\frac{T}{2 \pi} \log \left(1+\frac{h}{n}\right)\right) \\
& +O\left(T^{-1} N^{1-2 \sigma}\right) \\
= & \sum_{N<n} \sum_{1 \leq h<n / \tau} a_{n} \bar{b}_{n+h} K_{\sigma}(n, h)+O\left(T^{-1} N^{1-2 \sigma}\right),
\end{aligned}
$$

say. Changing the order of summation, which is permissible by absolute 
convergence, and then splitting the sum over $h$ at $H^{*}$, we obtain

$$
\begin{aligned}
I_{12}^{*}= & \sum_{1 \leq h \leq H^{*}} \sum_{\max (N, h \tau)<n} a_{n} \bar{b}_{n+h} K_{\sigma}(n, h)+\sum_{H^{*} \leq h} \sum_{h \tau<n} a_{n} \bar{b}_{n+h} K_{\sigma}(n, h) \\
& +O\left(T^{-1} N^{1-2 \sigma}\right) .
\end{aligned}
$$

By $\left(\mathrm{A}_{1}\right),(20),(37 \mathrm{~b})$, and $(29)$ the second term is

$$
\begin{aligned}
& \ll \sum_{H^{*}<h} \sum_{h \tau<n} n^{-2 \sigma+(\varepsilon / 4)(2 \sigma-2)} \ll \sum_{H^{*}<h}(h \tau)^{1-2 \sigma+(\varepsilon / 2)(2 \sigma-2)} \\
& \ll \tau^{1-2 \sigma+\varepsilon(2 \sigma-2)}\left(H^{*}\right)^{(2-2 \sigma)(1-\varepsilon)} \\
& =\tau^{-\varepsilon} N^{1-2 \sigma} \ll T^{-\varepsilon / 2} N^{1-2 \sigma} .
\end{aligned}
$$

Thus, by (9) and Stieltjes integration we have

$$
\begin{aligned}
I_{12}^{*}= & \sum_{1 \leq h \leq H^{*}} \int_{\max (N, h \tau)}^{\infty} M_{1}^{\prime}(x, h) K_{\sigma}(x, h) d x \\
& +\sum_{1 \leq h \leq H^{*} \max (N, h \tau)} \int_{\sigma}^{\infty} K_{\sigma}(x, h) d E_{1}(x, h)+O\left(T^{-\varepsilon / 2} N^{1-2 \sigma}\right) .
\end{aligned}
$$

The second term equals

$$
\begin{aligned}
\sum_{1 \leq h \leq H^{*}}\left(\left.E_{1}(x, h) K_{\sigma}(x, h)\right|_{\max (N, h \tau)} ^{\infty}\right. & \\
& \left.-\int_{\max (N, h \tau)}^{\infty} E_{1}(x, h) \frac{\partial}{\partial x} K_{\sigma}(x, h) d x\right) .
\end{aligned}
$$

We may replace $E_{1}(x, h)$ here by $O\left(x^{\varphi}\right)$ if we can show that $h \leq x^{\eta}$ for all $1 \leq h \leq H^{*}$ and $x>\max (N, h \tau)$. This condition will be met if $H^{*} \leq$ $\tau^{\eta /(1-\eta)}$. But this follows immediately from (28) and (29), so we find that (51) is

$$
\begin{aligned}
& \ll T^{\varepsilon} \sum_{1 \leq h \leq H^{*}}(\max (N, h \tau))^{\varphi-2 \sigma} \\
& =T^{\varepsilon} \sum_{1 \leq h \leq N / \tau} N^{\varphi-2 \sigma}+T^{\varepsilon} \sum_{N / \tau<h \leq H^{*}}(h \tau)^{\varphi-2 \sigma} \\
& \ll T^{\varepsilon} \tau^{-1} N^{1-2 \sigma+\varphi} \ll T^{-1} N^{1-2 \sigma+\varphi+4 \varepsilon}
\end{aligned}
$$

by (20), (21), and (37b). Combining our estimates, we see that

$$
\begin{aligned}
I_{12}^{*}= & \sum_{1 \leq h \leq H^{*}} \int_{\max (N, h \tau)}^{\infty} M_{1}^{\prime}(x, h) K_{\sigma}(x, h) d x+O\left(T^{-1} N^{1-2 \sigma+\varphi+4 \varepsilon}\right) \\
& +O\left(T^{-\varepsilon / 2} N^{1-2 \sigma}\right) .
\end{aligned}
$$


Treating $I_{13}^{*}$ in the same way, we obtain

$$
\begin{aligned}
I_{13}^{*}= & \sum_{1 \leq h \leq H^{*}} \int_{\max (N, h \tau)}^{\infty} M_{2}^{\prime}(x, h) K_{\sigma}(x, h) d x+O\left(T^{-1} N^{1-2 \sigma+\varphi+4 \varepsilon}\right) \\
& +O\left(T^{-\varepsilon / 2} N^{1-2 \sigma}\right) .
\end{aligned}
$$

Hence, by (50) we have

$$
\begin{aligned}
I_{1}^{*}= & \widehat{\Psi}_{U}(0) T \sum_{N<n} a_{n} \bar{b}_{n} n^{-2 \sigma} \\
& +T \sum_{1 \leq h \leq H^{*}} \int_{\max (N, h \tau)}^{\infty} M_{1}^{\prime}(x, h) K_{\sigma}(x, h) d x \\
& +T \sum_{1 \leq h \leq H^{*}} \int_{\max (N, h \tau)}^{\infty} \overline{M_{2}^{\prime}(x, h) K_{\sigma}(x, h)} d x \\
& +O\left(N^{1-2 \sigma+\varphi+4 \varepsilon}\right)+O\left(T^{1-\varepsilon / 2} N^{1-2 \sigma}\right) .
\end{aligned}
$$

Next consider $I_{2}^{*}$. By (15) and absolute convergence we have

$$
\begin{aligned}
I_{2}^{*} & =T \sum_{N<n} a_{n} n^{-\sigma} \int_{N}^{\infty} \overline{M_{2}^{\prime}(x)} x^{-\sigma} \widehat{\Psi}_{U}\left(\frac{T}{2 \pi} \log \frac{x}{n}\right) d x \\
& =T \sum_{N<n} a_{n} n^{-\sigma}\left(\int_{n}^{\infty}+\int_{N}^{n}\right) \overline{M_{2}^{\prime}(x)} x^{-\sigma} \widehat{\Psi}_{U}\left(\frac{T}{2 \pi} \log \frac{x}{n}\right) d x
\end{aligned}
$$

or

$$
I_{2}^{*}=T I_{21}^{*}+T I_{22}^{*} .
$$

In $I_{21}^{*}$ we write $x=n+u$ and use $\left(\mathrm{A}_{1}\right),(6)$, and (19) for $u \geq n / \tau$ to obtain

$$
\begin{aligned}
I_{21}^{*}= & \sum_{N<n} a_{n} n^{-\sigma} \int_{0}^{n / \tau} \overline{M_{2}^{\prime}(n+u)}(n+u)^{-\sigma} \widehat{\Psi}_{U}\left(\frac{T}{2 \pi} \log \left(1+\frac{u}{n}\right)\right) d u \\
& +O\left(T^{-1} N^{1-2 \sigma}\right) \\
= & \sum_{N<n} a_{n} \int_{0}^{n / \tau} \overline{M_{2}^{\prime}(n+u)} K_{\sigma}(n, u) d u+O\left(T^{-1} N^{1-2 \sigma}\right),
\end{aligned}
$$

say. Changing the order of summation and integration (by absolute convergence) and then splitting the $u$ integral at $H^{*}$, we find that 


$$
\begin{aligned}
I_{21}^{*}= & \int_{0}^{H^{*}} \sum_{\max (N, u \tau)<n} a_{n} \overline{M_{2}^{\prime}(n+u)} K_{\sigma}(n, u) d u \\
& +\int_{H^{*}}^{\infty} \sum_{u \tau<n} a_{n} \overline{M_{2}^{\prime}(n+u)} K_{\sigma}(n, u) d u+O\left(T^{-1} N^{1-2 \sigma}\right) .
\end{aligned}
$$

By $\left(\mathrm{A}_{1}\right),(6),(20),(37 \mathrm{~b})$, and (29), the second term is

$$
\begin{aligned}
& \ll \int_{H^{*}}^{\infty}\left(\sum_{u \tau<n} n^{-2 \sigma+(\varepsilon / 4)(2 \sigma-2)}\right) d u \ll \int_{H^{*}}^{\infty}(u \tau)^{1-2 \sigma+(\varepsilon / 2)(2 \sigma-2)} d u \\
& \ll \tau^{1-2 \sigma+\varepsilon(2 \sigma-2)}\left(H^{*}\right)^{(2-2 \sigma)(1-\varepsilon)} \\
& =\tau^{-\varepsilon} N^{1-2 \sigma} \ll T^{-\varepsilon / 2} N^{1-2 \sigma} .
\end{aligned}
$$

Thus, by (4) and Stieltjes integration, we have

$$
\begin{aligned}
I_{21}^{*}= & \int_{0}^{H^{*}} \int_{\max (N, u \tau)}^{\infty} M_{1}^{\prime}(x) \overline{M_{2}^{\prime}(x+u)} K_{\sigma}(x, u) d x d u \\
& +\int_{0}^{H^{*}} \int_{\max (N, u \tau)^{-}}^{\infty} \overline{M_{2}^{\prime}(x+u)} K_{\sigma}(x, u) d E_{1}(x) d u \\
& +O\left(T^{-\varepsilon / 2} N^{1-2 \sigma}\right) .
\end{aligned}
$$

The second term is

$$
\begin{aligned}
\int_{0}^{H^{*}}\left(\overline{M_{2}^{\prime}(x+u)} K_{\sigma}(x, u)\right. & \left.\left.E_{1}(x)\right|_{\max (N, u \tau)^{-}} ^{\infty}\right) d u \\
& -\int_{0}^{H^{*}} \int_{\max (N, u \tau)}^{\infty}\left(\overline{M_{2}^{\prime \prime}(x+u)} K_{\sigma}(x, u)\right. \\
& \left.+\overline{M_{2}^{\prime}(x, u)} \frac{\partial}{\partial x} K_{\sigma}(x, u)\right) E_{1}(x) d x d u \\
& \ll T^{\varepsilon} \int_{0}^{H^{*}}(\max (N, u \tau))^{\theta-2 \sigma+\varepsilon / 2} d u \\
& \ll T^{\varepsilon} \int_{0}^{N / \tau} N^{\theta-2 \sigma+\varepsilon / 2} d u+T^{\varepsilon} \int_{N / \tau}^{H^{*}}(u \tau)^{\theta-2 \sigma+\varepsilon / 2} d u \\
& \ll T^{\varepsilon} \tau^{-1} N^{1-2 \sigma+\theta+\varepsilon} \ll T^{-1} N^{1-2 \sigma+\theta+5 \varepsilon}
\end{aligned}
$$


by $\left(\mathrm{A}_{2}\right),(20),(21)$, and (37a) and (37b). Thus we find that

$$
\begin{aligned}
I_{21}^{*}= & \int_{0}^{H^{*}} \int_{\max (N, u \tau)}^{\infty} M_{1}^{\prime}(x) \overline{M_{2}^{\prime}(x+u)} K_{\sigma}(x, u) d x d u \\
& +O\left(T^{-1} N^{1-2 \sigma+\theta+t \varepsilon}\right)+O\left(T^{-\varepsilon / 2} N^{1-2 \sigma}\right) .
\end{aligned}
$$

In $I_{22}^{*}$ we set $x=n-u$ and obtain

$$
I_{22}^{*}=\sum_{N<n} a_{n} n^{-\sigma} \int_{0}^{n-N} \overline{M_{2}^{\prime}(n-u)}(n-u)^{-\sigma} \widehat{\Psi}_{U}\left(\frac{T}{2 \pi} \log \left(1-\frac{u}{n}\right)\right) d u .
$$

By (19) for $u>n /(\tau+1)$, this equals

$$
\begin{aligned}
& \sum_{N<n} a_{n} n^{-\sigma} \int_{0}^{\min (n-N, n /(\tau+1))} \overline{M_{2}^{\prime}(n-u)}(n-u)^{-\sigma} \widehat{\Psi}_{U}\left(\frac{T}{2 \pi} \log \left(1-\frac{u}{n}\right)\right) d u+O\left(T^{-1} N^{1-2 \sigma}\right) \\
&=\sum_{N<n} a_{n} \int_{0}^{\min (n-N, n /(\tau+1))} \overline{M_{2}^{\prime}(n-u) K_{\sigma}(n-u, u)} d u+O\left(T^{-1} N^{1-2 \sigma}\right) .
\end{aligned}
$$

As in $I_{21}^{*}$ we interchange the order of summation and integration and split the resulting integral at $H^{*}$ to obtain

$$
\begin{aligned}
I_{22}^{*}= & \int_{0}^{H^{*}} \sum_{\max (N+u, u(\tau+1))<n} a_{n} \overline{M_{2}^{\prime}(n-u) K_{\sigma}(n-u, u)} d u \\
& +\int_{H^{*}}^{\infty} \sum_{u(\tau+1)<n} a_{n} \overline{M_{2}^{\prime}(n-u) K_{\sigma}(n-u, u)} d u+O\left(T^{-1} N^{1-2 \sigma}\right) .
\end{aligned}
$$

Estimating the second term as we did in the case of $I_{21}^{*}$, we see that it is $\ll T^{-\varepsilon / 2} N^{1-2 \sigma}$. Thus, by (4) and Stieltjes integration

$$
\begin{aligned}
I_{22}^{*}= & \int_{0}^{H^{*}} \int_{\max (N+u, u(\tau+1))}^{\infty} M_{1}^{\prime}(y) \overline{M_{2}^{\prime}(y-u) K_{\sigma}(y-u, u)} d y d u \\
& +\int_{0}^{H^{*}} \int_{\max (N+u, u(\tau+1))}^{\infty} \overline{M_{2}^{\prime}(y-u) K_{\sigma}(y-u, u)} d E_{1}(y) d u \\
& +O\left(T^{-\varepsilon / 2} N^{1-2 \sigma}\right) .
\end{aligned}
$$

The second term is estimated just like the corresponding term in (54) with the result that it also is $\ll T^{-1} N^{1-2 \sigma+\theta+5 \varepsilon}$. In the first term we make the 
substitution $x=y-u$ and find that

$$
\begin{aligned}
I_{22}^{*}= & \int_{0}^{H^{*}} \int_{\max (N, u \tau)}^{\infty} \overline{M_{2}^{\prime}(x)} M_{1}^{\prime}(x+u) \overline{K_{\sigma}(x, u)} d x d u \\
& +O\left(T^{-1} N^{1-2 \sigma+\theta+5 \varepsilon}\right)+O\left(T^{-\varepsilon / 2} N^{1-2 \sigma}\right) .
\end{aligned}
$$

Combining (53), (55), and (56), we now have

$$
\begin{aligned}
I_{2}^{*}= & T \int_{0}^{H^{*}} \int_{\max (N, u \tau)}^{\infty} M_{1}^{\prime}(x+u) \overline{M_{2}^{\prime}(x) K_{\sigma}(x, u)} d x d u \\
& +T \int_{0}^{H^{*}} \int_{\max (N, u \tau)}^{\infty} \overline{M_{2}^{\prime}(x+u)} M_{1}^{\prime}(x) K_{\sigma}(x, u) d x d u \\
& +O\left(N^{1-2 \sigma+\theta+5 \varepsilon}\right)+O\left(T^{1-\varepsilon / 2} N^{1-2 \sigma}\right) .
\end{aligned}
$$

Since $I_{3}^{*}$ is the complex conjugate of $I_{2}^{*}$ with $\mathbb{B}^{*}(s)$ replacing $\mathbb{A}^{*}(s)$ and $M_{1}^{\prime}(x)$ replacing $M_{2}^{\prime}(x)$, it follows from (57) that

$$
\begin{aligned}
I_{3}^{*}= & T \int_{0}^{H^{*}} \int_{\max (N, u \tau)}^{\infty} \overline{M_{2}^{\prime}(x+u)} M_{1}^{\prime}(x) K_{\sigma}(x, u) d x d u \\
& +T \int_{0}^{H^{*}} \int_{\max (N, u \tau)}^{\infty} M_{1}^{\prime}(x+u) \overline{M_{2}^{\prime}(x) K_{\sigma}(x, u)} d x d u \\
& +O\left(N^{1-2 \sigma+\theta+5 \varepsilon}\right)+O\left(T^{1-\varepsilon / 2} N^{1-2 \sigma}\right) .
\end{aligned}
$$

Note that this is identical to the expression for $I_{2}^{*}$.

Next we treat $I_{4}^{*}$. By absolute convergence, (15), and (16) we have

$$
\begin{aligned}
I_{4}^{*}= & T \int_{N}^{\infty} \int_{x}^{\infty} M_{1}^{\prime}(x) \overline{M_{2}^{\prime}(y)}(x y)^{-\sigma} \widehat{\Psi}_{U}\left(\frac{T}{2 \pi} \log \frac{y}{x}\right) d y d x \\
& +T \int_{N}^{\infty} \int_{x}^{\infty} M_{2}^{\prime}(x) \overline{M_{1}^{\prime}(y)}(x y)^{-\sigma} \widehat{\Psi}_{U}\left(\frac{T}{2 \pi} \log \frac{y}{x}\right) d y d x \\
= & T I_{41}^{*}+T \overline{I_{42}^{*}},
\end{aligned}
$$

say. In $I_{41}^{*}$ we set $y=x+u$ and use (6) and (19) for $u>x / \tau$ to obtain

$$
\begin{aligned}
I_{41}^{*}= & \int_{N}^{\infty} \int_{0}^{x / \tau} M_{1}^{\prime}(x) \overline{M_{2}^{\prime}(x+u)} x^{-2 \sigma}\left(1+\frac{u}{x}\right)^{-\sigma} \widehat{\Psi}_{U}\left(\frac{T}{2 \pi} \log \left(1+\frac{u}{x}\right)\right) d u d x \\
& +O\left(T^{-1} N^{1-2 \sigma}\right)
\end{aligned}
$$




$$
=\int_{N}^{\infty} \int_{0}^{x / \tau} M_{1}^{\prime}(x) \overline{M_{2}^{\prime}(x+u)} K_{\sigma}(x, u) d u d x+O\left(T^{-1} N^{1-2 \sigma}\right) .
$$

The double integral converges absolutely so we may change the order of integration. After doing so and splitting the resulting $u$ integral at $H^{*}$, we obtain

$$
\begin{aligned}
I_{41}^{*}= & \int_{0}^{H^{*}} \int_{\max (N, u \tau)}^{\infty} M_{1}^{\prime}(x) \overline{M_{2}^{\prime}(x+u)} K_{\sigma}(x, u) d x d u \\
& +\int_{H^{*}}^{\infty} \int_{u \tau}^{\infty} M_{1}^{\prime}(x) \overline{M_{2}^{\prime}(x+u)} K_{\sigma}(x, u) d x d u+O\left(T^{-1} N^{1-2 \sigma}\right) .
\end{aligned}
$$

By (6), (20), (37a), and (29), the second term is

$$
\begin{aligned}
& \ll \int_{H^{*}}^{\infty} \int_{u \tau}^{\infty} x^{-2 \sigma+(\varepsilon / 4)(2 \sigma-2)} d x d u \ll \int_{H^{*}}^{\infty}(u \tau)^{1-2 \sigma+(\varepsilon / 2)(2 \sigma-2)} d u \\
& \ll \tau^{1-2 \sigma+\varepsilon(2 \sigma-2)}\left(H^{*}\right)^{(2-2 \sigma)(1-\varepsilon)} \\
& \ll \tau^{-\varepsilon} N^{1-2 \sigma} \ll T^{-\varepsilon / 2} N^{1-2 \sigma} .
\end{aligned}
$$

Thus we find that

$$
I_{41}^{*}=\int_{0}^{H^{*}} \int_{\max (N, u \tau)}^{\infty} M_{1}^{\prime}(x) \overline{M_{2}^{\prime}(x+u)} K_{\sigma}(x, u) d x d u+O\left(T^{-\varepsilon / 2} N^{1-2 \sigma}\right) .
$$

Since $I_{42}^{*}$ is $I_{41}^{*}$ with $M_{1}^{\prime}$ and $M_{2}^{\prime}$ interchanged, we see that

$$
I_{42}^{*}=\int_{0}^{H^{*}} \int_{\max (N, u \tau)}^{\infty} M_{2}^{\prime}(x) \overline{M_{1}^{\prime}(x+u)} K_{\sigma}(x, u) d x d u+O\left(T^{-\varepsilon / 2} N^{1-2 \sigma}\right) .
$$

Thus, we find that

$$
\begin{aligned}
I_{4}^{*}= & T \int_{0}^{H^{*}} \int_{\max (N, u \tau)}^{\infty} M_{1}^{\prime}(x+u) \overline{M_{2}^{\prime}(x) K_{\sigma}(x, u)} d x d u \\
& +T \int_{0}^{H^{*}} \int_{\max (N, u \tau)}^{\infty} \overline{M_{2}^{\prime}(x+u)} M_{1}^{\prime}(x) K_{\sigma}(x, u) d x d u \\
& +O\left(T^{1-\varepsilon / 2} N^{1-2 \sigma}\right) .
\end{aligned}
$$

By (49), (52), (57), (58), and (59), we now see that 
$(60)$

$$
\begin{aligned}
I^{*}= & \widehat{\Psi}_{U}(0) T \sum_{N<n} a_{n} \bar{b}_{n} n^{-2 \sigma} \\
& +T \sum_{1 \leq h \leq H^{*}} \int_{\max (N, h \tau)}^{\infty} M_{1}^{\prime}(x, h) K_{\sigma}(x, h) d x \\
& +T \sum_{1 \leq h \leq H^{*}} \int_{\max (N, h \tau)}^{\infty} \overline{M_{2}^{\prime}(x, h) K_{\sigma}(x, h)} d x \\
& -T \int_{0}^{H^{*}} \int_{\max (N, u \tau)}^{\infty} M_{1}^{\prime}(x+u) \overline{M_{2}^{\prime}(x) K_{\sigma}(x, u)} d x d u \\
& -T \int_{0}^{H^{*}} \int_{\max (N, u \tau)}^{\infty} \overline{M_{2}^{\prime}(x+u)} M_{1}^{\prime}(x) K_{\sigma}(x, u) d x d u \\
& +O\left(N^{1-2 \sigma+\max (\theta, \varphi)+5 \varepsilon}\right)+O\left(T^{1-\varepsilon / 2} N^{1-2 \sigma}\right) .
\end{aligned}
$$

This is (30) so the proof of Theorem 2 is complete.

The proof of Corollary 2 is along the same lines as that of Corollary 1 so we leave out most of the details. Replacing $K_{\sigma}(x, u)$ by $x^{-2 \sigma} \widehat{\Psi}_{U}\left(\frac{u T}{2 \pi x}\right)$ and $M_{i}^{\prime}(x+u)$ by $M_{i}^{\prime}(x)(i=1,2)$, we see from (23), (48), and the fact that $\sigma>1$ and $N \gg T$, that the right-hand side of (60) changes by no more than $O\left(T^{-1} N^{2-2 \sigma+5 \varepsilon}\right)$. Therefore we have

$$
\begin{aligned}
I^{*}= & \widehat{\Psi}_{U}(0) \sum_{N<n} a_{n} \bar{b}_{n} n^{-2 \sigma} \\
& +T \sum_{1 \leq h \leq H^{*}} \int_{\max (N, h \tau)}^{\infty} M_{1}^{\prime}(x, h) x^{-2 \sigma} \widehat{\Psi}_{U}\left(\frac{h T}{2 \pi x}\right) d x \\
& +T \sum_{1 \leq h \leq H^{*}} \int_{\max (N, h \tau)}^{\infty} \overline{M_{2}^{\prime}(x, h)} x^{-2 \sigma} \overline{\widehat{\Psi}_{U}\left(\frac{h T}{2 \pi x}\right)} d x \\
& -2 T \int_{0}^{H_{\max }^{*}} \int_{N, u \tau)}^{\infty} M_{1}^{\prime}(x) \overline{M_{2}^{\prime}(x)} x^{-2 \sigma} \operatorname{Re} \widehat{\Psi}_{U}\left(\frac{u T}{2 \pi x}\right) d x d u \\
& +O\left(N^{1-2 \sigma+\max (\theta, \varphi)+5 \varepsilon}\right)+O\left(T^{1-\varepsilon / 2} N^{1-2 \sigma}\right) \\
& +O\left(T^{-1} N^{2-2 \sigma+5 \varepsilon}\right) .
\end{aligned}
$$

Next consider the second term on the right-hand side of (61). If we replace the lower limit of integration by $N$, then this changes the term by the amount

$$
T \sum_{N / \tau<h \leq H^{*}} \int_{N}^{h \tau} M_{1}^{\prime}(x, h) x^{-2 \sigma} \widehat{\Psi}_{U}\left(\frac{h T}{2 \pi x}\right) d x .
$$


For this range of $h$ and $x$ we have $h /(2 \pi x) \gg \tau^{-1}$, so by (19) this term is negligible. We may therefore take the second term to be

$$
T \sum_{1 \leq h \leq H^{*}} \int_{N}^{\infty} M_{1}^{\prime}(x, h) x^{-2 \sigma} \widehat{\Psi}_{U}\left(\frac{h T}{2 \pi x}\right) d x .
$$

We now set $v=h T /(2 \pi x)$ and find that this equals

$$
C_{T} \sum_{1 \leq h \leq H^{*}} h^{1-2 \sigma} \int_{0}^{h T /(2 \pi N)} M_{1}^{\prime}\left(\frac{h T}{2 \pi v}, h\right) v^{2 \sigma-2} \widehat{\Psi}_{U}(v) d v .
$$

Changing the order of summation and integration, we find that this is

$$
\begin{aligned}
C_{T} & \int_{0}^{T /(2 \pi N)}\left(\sum_{1 \leq h \leq H^{*}} M_{1}^{\prime}\left(\frac{h T}{2 \pi v}, h\right) h^{1-2 \sigma}\right) \widehat{\Psi}_{U}(v) v^{2 \sigma-2} d v \\
& +C_{T} \int_{T /(2 \pi N)}^{T H^{*} /(2 \pi N)}\left(\sum_{2 \pi N v / T<h \leq H^{*}} M_{1}^{\prime}\left(\frac{h T}{2 \pi v}, h\right) h^{1-2 \sigma}\right) \widehat{\Psi}_{U}(v) v^{2 \sigma-2} d v .
\end{aligned}
$$

Similarly, the third term on the right-hand side of (61) is

$$
\begin{aligned}
& C_{T} \int_{0}^{T /(2 \pi N)}\left(\sum_{1 \leq h \leq H^{*}} \overline{M_{2}^{\prime}\left(\frac{h T}{2 \pi v}, h\right)} h^{1-2 \sigma)} \overline{\widehat{\Psi}_{U}(v)} v^{2 \sigma-2} d v\right. \\
& +C_{T} \int_{T /(2 \pi N)}^{T H^{*} /(2 \pi N)}\left(\sum_{2 \pi N v / T<h \leq H^{*}} \overline{M_{2}^{\prime}\left(\frac{h T}{2 \pi v}, h\right)} h^{1-2 \sigma)} \overline{\widehat{\Psi}_{U}(v)} v^{2 \sigma-2} d v .\right.
\end{aligned}
$$

Finally, the same basic analysis applied to the fourth term on the right-hand side of (61) leads to

$-2 C_{T} \int_{0}^{H^{*} T /(2 \pi N)}\left(\int_{2 \pi N v / T}^{H^{*}} M_{1}^{\prime}\left(\frac{u T}{2 \pi v}\right) \overline{M_{2}^{\prime}\left(\frac{u T}{2 \pi v}\right)} u^{1-2 \sigma} d u\right) \operatorname{Re} \widehat{\Psi}_{U}(v) v^{2 \sigma-2} d v$.

Combining our expressions, we obtain (31), so the proof of Corollary 2 is complete.

4. Proof of Theorem 3. We have

$$
\begin{aligned}
J= & \int_{-\infty}^{\infty} \Psi_{U}\left(\frac{t}{T}\right) \\
& \times\left(\mathbb{A}(s)-\int_{1}^{N} M_{1}^{\prime}(x) x^{-s} d x\right) \overline{\left(\mathbb{B}^{*}\left(s^{\prime}\right)-\int_{N}^{\infty} M_{2}^{\prime}(x) x^{-s^{\prime}} d x\right)} d t
\end{aligned}
$$




$$
\begin{aligned}
= & \int_{-\infty}^{\infty} \Psi_{U}\left(\frac{t}{T}\right) \mathbb{A}(s) \overline{\mathbb{B}^{*}\left(s^{\prime}\right)} d t \\
& -\int_{-\infty}^{\infty} \Psi_{U}\left(\frac{t}{T}\right) \mathbb{A}(s) \overline{\left(\int_{N}^{\infty} M_{2}^{\prime}(x) x^{-s^{\prime}} d x\right)} d t \\
& -\int_{-\infty}^{\infty} \Psi_{U}\left(\frac{t}{T}\right) \overline{\mathbb{B}^{*}\left(s^{\prime}\right)}\left(\int_{1}^{N} M_{1}^{\prime}(x) x^{-s} d x\right) d t \\
& +\int_{-\infty}^{\infty} \Psi_{U}\left(\frac{t}{T}\right)\left(\int_{1}^{N} M_{1}^{\prime}(x) x^{-s} d x\right) \overline{\left(\int_{N}^{\infty} M_{2}^{\prime}(x) x^{-s^{\prime}} d x\right)} d t \\
= & J_{1}-J_{2}-J_{3}+J_{4},
\end{aligned}
$$

say. By (15) we see that

$$
J_{1}=T \sum_{n \leq N} \sum_{N<m} a_{n} \bar{b}_{m} n^{-\sigma} m^{-\sigma^{\prime}} \widehat{\Psi}_{U}\left(\frac{T}{2 \pi} \log \frac{m}{n}\right) .
$$

Now if either $(m-N) / N$ or $(N-n) / N$ is greater than $\tau^{-1}$, then $\log (m / n)$ $\gg \tau^{-1}$ so that by (19),

$$
\widehat{\Psi}_{U}\left(\frac{T}{2 \pi} \log \frac{m}{n}\right) \ll T^{-D_{2}} \quad \text { for any } D_{2} .
$$

Hence, we have

$$
\begin{aligned}
J_{1}= & T \sum_{N\left(1-\tau^{-1}\right) \leq n \leq N} \sum_{N<m \leq N\left(1+\tau^{-1}\right)} a_{n} \bar{b}_{m} n^{-\sigma} m^{-\sigma^{\prime}} \widehat{\Psi}_{U}\left(\frac{T}{2 \pi} \log \frac{m}{n}\right) \\
& +O\left(T^{-1} N^{2-\sigma-\sigma^{\prime}}\right),
\end{aligned}
$$

say. By $\left(A_{1}\right),(17)$, and $(37 b)$, this is

$$
\begin{aligned}
& \ll T \sum_{N(1-1 / \tau) \leq n \leq N} \sum_{N<m \leq N(1+1 / \tau)} n^{\varepsilon / 2-\sigma} m^{\varepsilon / 2-\sigma^{\prime}}+T^{-1} N^{2-\sigma-\sigma^{\prime}} \\
& \ll T \tau^{-2} N^{2-\sigma-\sigma^{\prime}+\varepsilon}+T^{-1} N^{2-\sigma-\sigma^{\prime}} \\
& \ll T^{-1+2 \varepsilon} N^{2-\sigma-\sigma^{\prime}+\varepsilon} \ll T^{-1} N^{2-\sigma-\sigma^{\prime}+5 \varepsilon} .
\end{aligned}
$$
Thus,

The integrals $J_{2}, J_{3}$, and $J_{4}$ are treated similarly with the same result.

$$
J \ll T^{-1} N^{2-\sigma-\sigma^{\prime}+5 \varepsilon}
$$

and the proof of the theorem is complete. 
5. Four examples. The following examples illustrate the application of some of our results.

ExAMPLE 1. Let $a_{n}=b_{n}=1$ for $n=1,2, \ldots$ Then

$$
A(x)=B(x)=C_{1}(x, h)=C_{2}(x, h)=[x]
$$

so that we may take

$$
M_{1}(x)=M_{2}(x)=M_{1}(x, h)=M_{2}(x, h)=x
$$

and

$$
E_{1}(x)=E_{2}(x)=E_{1}(x, h)=E_{2}(x, h) \ll 1 .
$$

In Corollary 1 we may therefore take $\theta=\varphi=0$ and $\eta=1-\varepsilon$, where $0<\varepsilon<1 / 2$ is arbitrarily small. Also, taking $U=\log T, N \gg T$, and $\sigma=1 / 2$, we find that

$$
\begin{aligned}
I= & \int_{-\infty}^{\infty} \Psi_{U}\left(\frac{t}{T}\right)\left|\sum_{n \leq N} n^{-1 / 2-i t}-\int_{1}^{N} x^{-1 / 2-i t} d x\right|^{2} d t \\
= & \widehat{\Psi}_{U}(0) T \sum_{n \leq N} n^{-1}+2 T \int_{T /(2 \pi N)}^{\infty}\left[\frac{2 \pi N}{T} v\right] \operatorname{Re} \widehat{\Psi}_{U}(v) v^{-1} d v \\
& -2 T \int_{T /(2 \pi N \tau)}^{\infty} \frac{2 \pi N}{T} v \operatorname{Re} \widehat{\Psi}_{U}(v) v^{-1} d v \\
& +O\left(T^{-1} N^{1+5 \varepsilon}\right) .
\end{aligned}
$$

Since $\widehat{\Psi}_{U}(v) \ll 1$, the lower limit of integration in the third term may be replaced by $T /(2 \pi N)$ with an error of $O(T)$. Thus we may rewrite the above as

$$
\begin{aligned}
I= & \widehat{\Psi}_{U}(0) T \log N-2 T \int_{T /(2 \pi N)}^{1}\left(\frac{2 \pi N}{T} v-\left[\frac{2 \pi N}{T} v\right]-\frac{1}{2}\right) \operatorname{Re} \widehat{\Psi}_{U}(v) v^{-1} d v \\
& -T \int_{T /(2 \pi N)}^{1} \operatorname{Re} \widehat{\Psi}_{U}(v) v^{-1} d v \\
& +2 T \int_{1}^{\infty}\left(\left[\frac{2 \pi N}{T} v\right]-\frac{2 \pi N}{T} v\right) \widehat{\Psi}_{U}(v) v^{-1} d v \\
& +O(T)+O\left(T^{-1} N^{1+5 \varepsilon}\right) .
\end{aligned}
$$

For $|v| \leq 1$ we have 


$$
\widehat{\Psi}_{U}(v)=\widehat{\Psi}_{U}(0)+O\left(\left(C_{2}-C_{1}+U^{-1}\right)|v|\right)
$$

and $\widehat{\Psi}_{U}(0)$ is real. Thus, the second term on the right-hand side above is

$$
\begin{aligned}
-2 \widehat{\Psi}_{U}(0) T \int_{T /(2 \pi N)}^{1} & \left(\frac{2 \pi N}{T} v-\left[\frac{2 \pi N}{T} v\right]-\frac{1}{2}\right) v^{-1} d v+O(T) \\
& =-2 \widehat{\Psi}_{U}(0) T \int_{1}^{2 \pi N / T}(y-[y]-1 / 2) y^{-1} d y+O(T) \\
& =O(T) .
\end{aligned}
$$

The third term equals

$$
-\widehat{\Psi}_{U}(0) T \int_{T /(2 \pi N)}^{1} v^{-1} d v+O(T)=\widehat{\Psi}_{U}(0) T \log (T / N)+O(T) .
$$

Finally, by (17) the fourth term is

$$
\ll T\left(\int_{1}^{U} v^{-1} d v+U \int_{U}^{\infty} v^{-2} d v\right) \ll T \log \log T .
$$

Thus we find that

$$
I=\widehat{\Psi}_{U}(0)(1+o(1)) T \log T+O\left(T^{-1} N^{1+5 \varepsilon}\right) .
$$

If in the definition of $\Psi_{U}(t)$ we take $C_{1}=U^{-1}$ and $C_{2}=1-U^{-1}$ and $\Psi_{U}(t) \leq 1$, then $\Psi_{U}(t)$ is a minorant for the characteristic function of the interval $[0,1]$. On the other hand, taking $C_{1}=-U^{-1}$ and $C_{2}=1+U^{-1}$, we obtain a majorant. Since in either case

$$
\widehat{\Psi}_{U}(0)=1+O\left(U^{-1}\right),
$$

it follows that

$$
\int_{0}^{T}\left|\sum_{n \leq N} n^{-1 / 2-i t}-\int_{1}^{N} x^{-1 / 2-i t} d x\right|^{2} d t \sim T \log T
$$

for $T \ll N \ll T^{2 /(1+5 \varepsilon)}$. Notice that if $N \ll T$, then the mean-square of $\int_{1}^{N} x^{-1 / 2-i t} d x$ is $\ll N \ll T$, so by (1) the left-hand side of (63) is $\sim T \log N$. We conclude this example by remarking that since $\theta=\varphi=0$ and $\eta=1-\varepsilon$, a straightforward but tedious application of Theorem 1 would allow us to prove that (63) is in fact valid for $T \ll N \ll T^{A}$ for any fixed $A \geq 1$. It is interesting to note that this and the simple approximation (see Titchmarsh $[5 ;$ p. 49]) 


$$
\begin{aligned}
\zeta\left(\frac{1}{2}+i t\right)= & \sum_{n \leq N} n^{-1 / 2-i t}-\int_{1}^{N} x^{-1 / 2-i t} d x \\
& +O\left(\left|\frac{1}{2}+i t\right| N^{-1 / 2}\right)+O\left(\left|\frac{1}{2}+i t\right|^{-1}\right),
\end{aligned}
$$

with $N=T^{2}$, gives the classical mean value formula

$$
\int_{0}^{T}\left|\zeta\left(\frac{1}{2}+i t\right)\right|^{2} d t \sim T \log T .
$$

EXAMPLE 2. Let $a_{n}=e(n \alpha), b_{n}=e(n \beta)$ for $n=1,2, \ldots$, with $0<$ $\alpha, \beta<1$.

Consider first the case where $\alpha=\beta$. Then

$$
A(x)=B(x)=\sum_{n \leq x} e(n \alpha) \ll 1,
$$

so we may take $M_{i}(x)=0$ and $E_{i}(x) \ll 1(i=1,2)$. Also,

$$
C_{i}(x, h)=e(-h \alpha)[x],
$$

so we may take $M_{i}(x, h)=e(-h \alpha) x$ and $E_{i}(x, h) \ll 1(i=1,2)$. Thus $\theta=\varphi=0$ and we may take $\eta=1-\varepsilon$. Taking $U=(\log T)^{2}, N \geq T$, and $\sigma=1 / 2$ in Corollary 1 , we find that

$$
\begin{aligned}
I= & \int_{-\infty}^{\infty} \Psi_{U}\left(\frac{t}{T}\right)\left|\sum_{n \leq N} e(n \alpha) n^{-1 / 2-i t}\right|^{2} d t \\
= & \widehat{\Psi}_{U}(0) T \sum_{n \leq N} n^{-1}+2 T \operatorname{Re} \int_{T /(2 \pi N)}^{\infty}\left(\sum_{1 \leq h \leq 2 \pi N v / T} e(-h \alpha)\right) \widehat{\Psi}_{U}(v) v^{-1} d v \\
& +O\left(T^{-1} N^{1+5 \varepsilon}\right) \\
= & \widehat{\Psi}_{U}(0) T(\log N+\gamma+O(1 / N)) \\
& -2 T \operatorname{Re} \int_{T /(2 \pi N)}^{\infty}\left(\frac{e\left(-\alpha\left[\frac{2 \pi N}{T} v\right]\right)-1}{e(\alpha)-1}\right) \widehat{\Psi}_{U}(v) v^{-1} d v \\
& +O\left(T^{-1} N^{1+5 \varepsilon}\right) .
\end{aligned}
$$

By (62) the middle term equals

$$
\begin{array}{r}
-2 \widehat{\Psi}_{U}(0) T \operatorname{Re}\left(\frac{1}{e(\alpha)-1} \int_{T /(2 \pi N)}^{1}\left(e\left(-\alpha\left[\frac{2 \pi N}{T} v\right]\right)-1\right) v^{-1} d v\right) \\
+O\left(\left(C_{2}-C_{1}+U^{-1}\right) T\right)+O\left(T \int_{1}^{\infty}\left|\widehat{\Psi}_{U}(v)\right| v^{-1} d v\right) .
\end{array}
$$


The second error term here is

$$
\begin{aligned}
& \ll\left(C_{2}-C_{1}+U^{-1}\right) T\left(\int_{1}^{U} v^{-1} d v+U \int_{U}^{\infty} v^{-2} d v\right) \\
& \ll\left(C_{2}-C_{1}+U^{-1}\right) T \log \log T
\end{aligned}
$$

by (17). Thus, changing variables in the remaining integral, we find that the middle term above equals

$$
\begin{aligned}
-2 \widehat{\Psi}_{U}(0) T \operatorname{Re}\left(\frac{1}{e(\alpha)-1} \int_{1}^{2 \pi N / T}(e(-\alpha[y])-1) y^{-1} d y\right) \\
+O\left(\left(C_{2}-C_{1}+U^{-1}\right) T \log \log T\right) .
\end{aligned}
$$

Now

$$
\begin{aligned}
& \int_{1}^{2 \pi N / T}(e(-\alpha[y])-1) y^{-1} d y \\
= & \sum_{k=1}^{[2 \pi N / T]-1} e(-\alpha k) \int_{k}^{k+1} y^{-1} d y+e\left(-\alpha\left[\frac{2 \pi N}{T}\right]\right) \log \frac{2 \pi N / T}{[2 \pi N / T]}-\log \frac{2 \pi N}{T} \\
= & \log (T / N)+O(1)
\end{aligned}
$$

by partial summation. Hence, our middle term is equal to

$$
2 \widehat{\Psi}_{U}(0) \operatorname{Re}\left(\frac{1}{e(\alpha)-1}\right) T \log (N / T)+O\left(\left(C_{2}-C_{1}+U^{-1}\right) T \log \log T\right) .
$$

Observing that $\operatorname{Re}(e(\alpha)-1)^{-1}=-1 / 2$ and combining our results, we find that

$$
\begin{aligned}
I=\widehat{\Psi}_{U}(0) T \log T+O\left(\left(C_{2}-C_{1}+U^{-1}\right) T \log \log T\right) \\
\quad+O\left(T^{-1} N^{1+5 \varepsilon}\right) .
\end{aligned}
$$

If we remove the weight function $\Psi_{U}(t / T)$ as in the last example, we deduce that

$$
\int_{0}^{T}\left|\sum_{n \leq N} e(n \alpha) n^{-1 / 2-i t}\right|^{2} d t \sim T \log T
$$

for $T \leq N \ll T^{2 /(1+5 \varepsilon)}$. Note that by (1) the left-hand side is $\sim T \log N$ when $N<T$. Had we used Theorem 1 rather than Corollary 1 , we could have shown (with more work) that (65) in fact holds for $T \leq N \ll T^{A}$ for any $A \geq 1$.

Now consider the case when $\alpha \neq \beta$. Here we may take

$$
M_{1}(x)=M_{2}(x)=M_{1}(x, h)=M_{2}(x, h)=0
$$


and

$$
E_{1}(x), E_{2}(x), E_{1}(x, h), E_{2}(x, h) \ll 1 .
$$

It is particularly easy to use Theorem 1 in such a case. We take $\theta=\varphi$ $=0, \eta=1-\varepsilon, \sigma=1 / 2, U=(\log T)^{2}$, and $T^{1-\varepsilon} \ll N \ll T^{(1-\varepsilon) /(5 \varepsilon)}$, and find that

$$
\begin{aligned}
I & =\int_{-\infty}^{\infty} \Psi_{U}\left(\frac{t}{T}\right)\left(\sum_{n \leq N} e(n \alpha) n^{-1 / 2-i t}\right)\left(\sum_{n \leq N} e(-n \beta) n^{-1 / 2+i t}\right) d t \\
& =\widehat{\Psi}_{U}(0) T \sum_{n \leq N} e(n(\alpha-\beta)) n^{-1}+O\left(N^{5 \varepsilon}\right) \\
& =-\widehat{\Psi}_{U}(0) \log (1-e(\alpha-\beta)) T+O\left(T^{1-\varepsilon}\right) .
\end{aligned}
$$

If we are interested in the unweighted integral

$$
I^{\prime}=\int_{0}^{T}\left(\sum_{n \leq N} e(n \alpha) n^{-1 / 2-i t}\right)\left(\sum_{n \leq N} e(-n \beta) n^{-1 / 2+i t}\right) d t
$$

instead, we can proceed as follows. We take $C_{1}=-U^{-1}$ and $C_{2}=1+U^{-1}$ in the definition of $\Psi_{U}$ in $I$ and use the Cauchy-Schwarz inequality to see that

$$
\begin{aligned}
I^{\prime}-I \ll & \left\{\left(\int_{-2 U^{-1}}^{0}+\int_{1}^{1+2 U^{-1}}\right)\left|\sum_{n \leq N} e(n \alpha) n^{-1 / 2-i t}\right|^{2} d t\right\}^{1 / 2} \\
& \times\left\{\left(\int_{-2 U^{-1}}^{0}+\int_{1}^{1+2 U^{-1}}\right)\left|\sum_{n \leq N} e(n \beta) n^{-1 / 2-i t}\right|^{2} d t\right\}^{1 / 2} .
\end{aligned}
$$

These integrals can be estimated by using (64) with $C_{1}=-3 U^{-1}, C_{2}=$ $3 U^{-1}$ in the definition of $\Psi_{U}$ and then with $C_{1}=1-3 U^{-1}, C_{2}=1+3 U^{-1}$. This gives

$$
I^{\prime}-I \ll U^{-1} T \log T=o(T)
$$

provided that $T \leq N \ll T^{(2-\varepsilon) /(1+5 \varepsilon)}$. Thus, for $\alpha \neq \beta$ and for the same range of $N$, we have

$$
\begin{aligned}
\int_{0}^{T}\left(\sum_{n \leq N} e(n \alpha) n^{-1 / 2-i t}\right)\left(\sum_{n \leq N} e(-n \beta) n^{-1 / 2+i t}\right) d t & \\
= & (1+o(1)) \log \left(\frac{1}{1-e(\alpha-\beta)}\right) T .
\end{aligned}
$$

Had we proved a longer-range version of (64) (by appealing to Theorem 1 instead of Corollary 1), (66) would also hold in such a range. 
EXAmPLE 3. Let $a_{n}=b_{n}=\mu(n)$ (the Möbius function) for $n=1,2, \ldots$ If the Riemann hypothesis is true, then

$$
A(x)=B(x)=\sum_{n \leq x} \mu(n) \ll x^{1 / 2+\varepsilon}
$$

for every $\varepsilon>0$. Thus we may take $M_{1}(x)=M_{2}(x)=0$ and $E_{1}(x)=$ $E_{2}(x) \ll x^{1 / 2+\varepsilon}$. Unfortunately, even assuming the Riemann hypothesis, no one has yet proven an estimate of the type

$$
C_{i}(x, h)=\sum_{n \leq x} \mu(n) \mu(n+h) \ll x^{\varphi} \quad(i=1,2)
$$

for any $\varphi<1$. A reasonable conjecture is that

$$
C_{i}(x, h) \ll x^{1 / 2+\varepsilon} \quad(i=1,2)
$$

uniformly for $1 \leq h \leq x^{1-\varepsilon}$. If this is so, we may take $M_{i}(x, h)=0$ and $E_{i}(x, h) \ll x^{1 / 2+\varepsilon}$ uniformly for $1 \leq h \leq x^{1-\varepsilon}$; hence we may set $\theta=\varphi=$ $1 / 2+\varepsilon$ and $\eta=1-\varepsilon$. By Theorem 1 with $\sigma=1 / 2$ and $U=\log T$ we then find that

$$
\begin{aligned}
I & =\int_{-\infty}^{\infty} \Psi_{U}\left(\frac{t}{T}\right)\left|\sum_{n \leq N} \mu(n) n^{-1 / 2-i t}\right|^{2} d t \\
& =\widehat{\Psi}_{U}(0) T \sum_{n \leq N} \mu^{2}(n) n^{-1}+O\left(N^{1 / 2+6 \varepsilon}\right)
\end{aligned}
$$

for $T^{1-\varepsilon} \ll N=o\left(T^{(1-\varepsilon) / \varepsilon}\right)$. Taking $\Psi_{U}(t)$ as in the first example, we then obtain

$$
\int_{0}^{T}\left|\sum_{n \leq N} \mu(n) n^{-1 / 2-i t}\right|^{2} d t \sim \frac{6}{\pi^{2}} T \log N
$$

uniformly for $T^{1-\varepsilon} \ll N \ll T^{2 /(1+12 \varepsilon)}$. This estimate also holds for $1 \leq$ $N \ll T^{1-\varepsilon}$ by (1). If we assume that the functions $E_{i}(x, h)$ behave independently for different $h$, we would expect, by (33), that the error term in (67) could be replaced by $O\left(T^{1 / 2} N^{6 \varepsilon}\right)$. Then (68) would hold uniformly for $1 \leq N \leq T^{A}$, where $A>0$ is arbitrary but fixed.

EXAMPLE 4. In his work on the pair correlation of zeros of the Riemann zeta-function, Montgomery [3] introduced the function

$$
F(x, T)=\sum_{0<\gamma, \gamma^{\prime} \leq T} x^{i\left(\gamma-\gamma^{\prime}\right)} w\left(\gamma-\gamma^{\prime}\right)
$$

where $\varrho=\beta+i \gamma$ is a zero of $\zeta(s)$ and $w(u)=4 /\left(4+u^{2}\right)$. Montgomery 
proved, assuming the Riemann hypothesis, that for $x \geq 1, T \geq 2$,

$$
\begin{aligned}
2 \pi F(x, T)= & \int_{0}^{T} \mid \frac{1}{x}\left(\mathbb{A}\left(\frac{1}{2}+i t\right)-\int_{1}^{x} u^{-1 / 2-i t} d u\right) \\
& +\left.x\left(\mathbb{A}^{*}\left(\frac{3}{2}+i t\right)-\int_{x}^{\infty} u^{-3 / 2-i t} d u\right)\right|^{2} d t \\
& +O\left(\frac{T \log ^{2} T}{x^{2}}\right)+O\left(\log ^{3} T\right),
\end{aligned}
$$

where

$$
\mathbb{A}(s)=\sum_{n \leq x} \frac{\Lambda(n)}{n^{s}} \quad \text { and } \quad \mathbb{A}^{*}(s)=\sum_{n>x} \frac{\Lambda(n)}{n^{s}} .
$$

For $1 \leq x \leq T$ one can apply standard mean value theorems to estimate the right-hand side of (69). When $\log T<x \leq T$ the diagonal terms dominate, but when $1 \leq x \leq \log T$ the first error term above is treated more carefully and contributes the main term. In this way Montgomery obtained an asymptotic formula for $F(x, T)$ in the range $1 \leq x \leq T$. In the range $x \geq T$ the off-diagonal terms come into play. Let $a_{n}=b_{n}=\Lambda(n)$ and assume the Riemann hypothesis. Then we have

$$
A(x)=B(x)=\sum_{n \leq x} \Lambda(n)=x+O\left(x^{1 / 2+\varepsilon}\right) .
$$

The twin prime conjecture asserts that

$$
C_{1}(x, h)=C_{2}(x, h)=\sum_{n \leq x} \Lambda(n) \Lambda(n+h)=\mathfrak{S}(h) x+E(x, h)
$$

where

$$
\mathfrak{S}(h)= \begin{cases}2 C \prod_{\substack{p \mid h \\ p>2}}\left(\frac{p-1}{p-2}\right) & \text { if } h \text { is even and } h \neq 0, \\ 0 & \text { if } h \text { is odd, }\end{cases}
$$

with

$$
C=\prod_{p>2}\left(1-\frac{1}{(p-1)^{2}}\right)
$$

and

$$
E(x, h)=o(x)
$$

for any fixed $h, 1 \leq h \leq x$. A stronger form of the conjecture is that

$$
E(x, h)=O\left(x^{1 / 2+\varepsilon}\right)
$$


uniformly for $1 \leq h \leq x$, and Montgomery [3] suggested that this form of the conjecture implies that

$$
F(x, T) \sim \frac{T}{2 \pi} \log T \quad \text { for } T \leq x \leq T^{2-\varepsilon} .
$$

He further conjectured that if the error terms $E(x, h)$ behave independently for different $h,(70)$ should hold for $T \leq x \leq T^{A}$ for any fixed $A>1$. J. Bolanz [1] was the first person to completely carry out the details of this argument in the range $T \leq x \leq T^{3 / 2-\varepsilon}$ and, in written communication, he has informed us that he can extend the range to $T \leq x \leq T^{2-\varepsilon}$. Bolanz's work is long and complicated, for the most part, because he does not smooth the integral in (69). With Corollaries 1 and 2 and Theorem 3 in hand, however, the proof can be greatly simplified and we sketch it here.

We assume the Riemann hypothesis and the strong form of the twin prime conjecture. It clearly suffices to obtain an asymptotic estimate for the integral in (69) weighted by the same functions $\Psi_{U}(t / T)$ used in the first example. Then, on multiplying out we see by Theorem 3 that the cross term is $\ll T^{-1} x^{2+1 / 2-3 / 2+\varepsilon}=x^{1+\varepsilon} T^{-1}$. Thus, for $x \geq T$, the (weighted) right-hand side of (69) equals

$$
\frac{1}{2 \pi x^{2}} I_{1}(x, T)+\frac{x^{2}}{2 \pi} I_{2}(x, T)+O\left(\frac{x^{1+\varepsilon}}{T}\right),
$$

where

$$
I_{1}=\int_{-\infty}^{\infty} \Psi_{U}\left(\frac{t}{T}\right)\left|\mathbb{A}\left(\frac{1}{2}+i t\right)-\int_{1}^{x} u^{-1 / 2-i t} d u\right|^{2} d t
$$

and

$$
I_{2}=\int_{-\infty}^{\infty} \Psi_{U}\left(\frac{t}{T}\right)\left|\mathbb{A}^{*}\left(\frac{3}{2}+i t\right)-\int_{x}^{\infty} u^{-3 / 2-i t} d u\right|^{2} d t
$$

To complete the proof of (70) we evaluate $I_{1}$ and $I_{2}$ and show that

$$
I_{1} \sim \frac{1}{2} T x^{2} \log T, \quad I_{2} \sim \frac{T}{2 x^{2}} \log T
$$

uniformly for $x \leq T \leq x^{2-\varepsilon}$.

Consider $I_{1}$. By our assumptions we see that $\left(\mathrm{A}_{1}\right)$ through $\left(\mathrm{A}_{4}\right)$ are satisfied with $\theta=\varphi=1 / 2-\varepsilon$ and $\eta=1-\varepsilon$. Therefore, by Corollary 1 , we have

$$
\begin{aligned}
I_{1}= & \widehat{\Psi}_{U}(0) T \sum_{n \leq x} n \Lambda^{2}(n) \\
& +4 \pi\left(\frac{T}{2 \pi}\right)^{3} \int_{T /(2 \pi x)}^{\infty}\left(\sum_{h \leq 2 \pi x v / T} \mathfrak{S}(h) h^{2}\right) \operatorname{Re} \widehat{\Psi}_{U}(v) \frac{d v}{v^{3}}
\end{aligned}
$$




$$
\begin{aligned}
& -4 \pi\left(\frac{T}{2 \pi}\right)^{3} \int_{T /(2 \pi \tau x)}^{\infty}\left(\int_{0}^{2 \pi x v / T} u^{2} d u\right) \operatorname{Re} \widehat{\Psi}_{U}(v) \frac{d v}{v^{3}} \\
& +O\left(\frac{x^{3+\varepsilon}}{T}\right)+O\left(x^{5 / 2+\varepsilon}\right)
\end{aligned}
$$

for $T \leq x$. Using the prime number theorem to evaluate the first sum and combining the two integrals, we obtain

$$
\begin{aligned}
I_{1}= & \frac{1}{2} T x^{2} \log x+4 \pi\left(\frac{T}{2 \pi}\right)^{3} \int_{T /(2 \pi x)}^{\infty} E\left(\frac{2 \pi x v}{T}\right) \operatorname{Re} \widehat{\Psi}_{U}(v) \frac{d v}{v^{3}} \\
& +O\left(\frac{x^{3+\varepsilon}}{T}\right)+O\left(x^{5 / 2+\varepsilon}\right),
\end{aligned}
$$

where $E(x)$ is defined by

$$
E(x)=\sum_{h \leq x} \mathfrak{S}(h) h^{2}-x^{3} / 3 .
$$

One can show that

$$
\int_{1}^{x} E(u) \frac{d u}{u^{3}}=-\frac{1}{4} \log x+O(1)
$$

and this estimate along with the properties of $\widehat{\Psi}_{U}(v)$ allows us to deduce that

$$
I_{1}=\frac{1}{2} T x^{2} \log T(1+o(1))+O\left(\frac{x^{3+\varepsilon}}{T}\right)+O\left(x^{5 / 2+\varepsilon}\right) .
$$

This gives the result for $I_{1}$ asserted in (71).

The evaluation of $I_{2}$ is similar except that one uses Corollary 2 in place of Corollary 1.

The above argument can be modified to obtain lower bounds for $F(x, T)$ in the range $T \leq x \leq T^{3 / 2-\varepsilon}$ assuming the Generalized Riemann Hypothesis. The key new idea is to approximate the coefficients $\Lambda(n)$ by related ones whose correlation sums $C(x, h)$ can be shown, assuming the Generalized Riemann Hypothesis, to satisfy precise estimates uniformly in certain ranges. The proof of this result will appear in joint work [2] with A. Özlük and C. Snyder. Some of the above argument in Example 4 is also joint work with Özlük and Snyder.

\section{References}

[1] J. Bolanz, Über die Montgomery'sche Paarvermutung, Diplomarbeit Universität Freiburg, 1987, 1-131.

[2] D. A. Goldston, S. M. Gonek, A. E. Özlük, and C. Snyder, On the pair correlation of the zeros of the Riemann zeta-function, to appear. 
[3] H. L. Montgomery, The pair correlation of zeros of the zeta function, in: Proc. Sympos. Pure Math. 24, Amer. Math. Soc., Providence, R.I., 1973, 181-193.

[4] H. L. Montgomery and R. C. Vaughan, The large sieve, Mathematika 20 (1973), 119-134.

[5] E. C. Titchmarsh, The Theory of the Riemann Zeta-Function, 2nd ed., revised by D. R. Heath-Brown, Clarendon Press, Oxford, 1986.

Department of Mathematics and Computer Science San Jose State University San Jose, California 95192 U.S.A.

E-mail: goldston@jupiter.sjsu.edu
Department of Mathematics University of Rochester Rochester, New York 14627 U.S.A.

E-mail: gonek@math.rochester.edu 